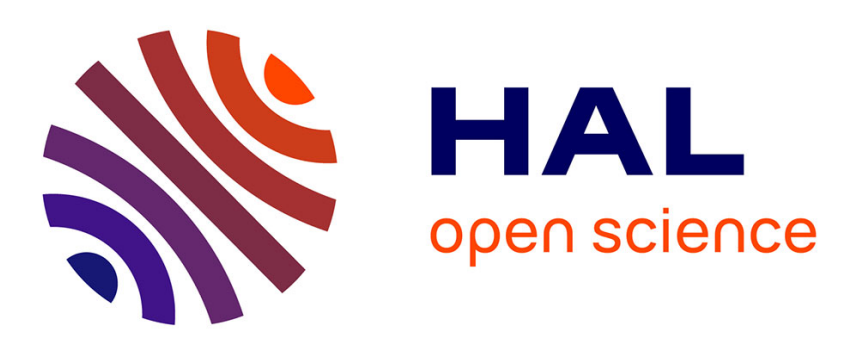

\title{
Collection of collapsing bubble driven phenomena found in champagne glasses
}

Gérard Liger-Belair, Thomas Séon, Arnaud Antkowiak

\section{To cite this version:}

Gérard Liger-Belair, Thomas Séon, Arnaud Antkowiak. Collection of collapsing bubble driven phenomena found in champagne glasses. Bubble Science, Engineering \& Technology, 2012, 4, pp.21 - 34. 10.1179/1758897912Y.0000000001?journalCode=ybub20 . hal-01447470

\section{HAL Id: hal-01447470 \\ https://hal.science/hal-01447470}

Submitted on 26 Jan 2017

HAL is a multi-disciplinary open access archive for the deposit and dissemination of scientific research documents, whether they are published or not. The documents may come from teaching and research institutions in France or abroad, or from public or private research centers.
L'archive ouverte pluridisciplinaire HAL, est destinée au dépôt et à la diffusion de documents scientifiques de niveau recherche, publiés ou non, émanant des établissements d'enseignement et de recherche français ou étrangers, des laboratoires publics ou privés. 


\title{
A collection of collapsing-bubble-driven phenomena found in champagne glasses
}

\author{
Gérard Liger-Belair ${ }^{1,2}$, Thomas Seon ${ }^{3}$, Arnaud Antkowiak ${ }^{3}$ \\ 1: Equipe Effervescence, Groupe de Spectrométrie Moléculaire et Atmosphérique (GSMA), \\ UMR CNRS 6089, UFR Sciences Exactes et Naturelles, BP 1039, 51687 Reims Cedex 2, \\ France.
}

2: Laboratoire d'Enologie et Chimie Appliquée, UPRES EA 2069, URVVC, UFR Sciences Exactes et Naturelles, Université de Reims, B.P. 1039, 51687 Reims Cedex 2, France.

3: Institut Jean Le Rond dAlembert (IJLRdA), UMR CNRS 7190, Université Pierre et Marie Curie, 4 Place Jussieu, 75252 Paris Cedex 05, France. 


\begin{abstract}
A simple glass of champagne or sparkling wine may seem like the acme of frivolity to most of people, but in fact it may rather be considered as a fantastic playground for any fluid physicist. In this tutorial review, the collapse of gaseous $\mathrm{CO}_{2}$ bubbles found at the free surface of a glass poured with champagne is depicted, through high-speed photography and highspeed video cameras. A collection of collapsing-bubble-driven phenomena are gathered, which illustrate the fine interplay between bubbles and the fluid around.
\end{abstract}




\section{Introduction}

Collapsing bubbles are very common in our everyday life. They play a crucial role in many natural as well as industrial processes (in physics, chemical and mechanical engineering, oceanography, geophysics, technology, and even medicine) ${ }^{1-5}$ Nevertheless, their behavior is often surprising and, in many cases, still not fully understood.

Since the end of the $17^{\text {th }}$ century, champagne has been a world-wide renowned French sparkling wine. Nevertheless, only quite recently, much interest has been devoted to characterize each and every parameter involved in its bubbling process. ${ }^{6}$ From a strictly physicochemical point of view, Champagne wines are multicomponent hydroalcoholic systems, with a density close to unity, a surface tension $\gamma \approx 50 \mathrm{mN} \mathrm{m}^{-1}$, and a viscosity about $50 \%$ larger than that of pure water. ${ }^{6}$ Champagne wines are also supersaturated with $\mathrm{CO}_{2}$ dissolved gas molecules formed together with ethanol during the second fermentation process (called prise de mousse, and promoted by adding yeasts and a certain amount of sugar inside bottles filled with a base wine and sealed with a cap). Actually, during this second fermentation process which occurs in cool cellars, the bottles are sealed, so that the $\mathrm{CO}_{2}$ molecules cannot escape and progressively dissolve into the wine. ${ }^{7-9}$ Therefore, $\mathrm{CO}_{2}$ molecules dissolved into the wine and gaseous $\mathrm{CO}_{2}$ molecules trapped under the cork progressively establish equilibrium (an application of Henry's law which states that the concentration of a given gas molecule dissolved into a solution is proportional to its partial pressure in the vapor phase, above the solution). Champagne wines therefore hold a concentration of dissolved $\mathrm{CO}_{2}$ proportional to the level of sugar added to promote this second fermentation. ${ }^{7-9}$ Actually, a standard 75 centiliters champagne bottle typically holds about 9 grams of dissolved $\mathrm{CO}_{2}$, which correspond to a volume close to 5 liters of gaseous $\mathrm{CO}_{2}$ under standard conditions for temperature and pressure. This volume of dissolved $\mathrm{CO}_{2}$ is 
responsible for bubble nucleation once the bottle is uncorked and the wine poured into a glass.

To get an idea of how many bubbles are potentially involved all along the degassing process from this single bottle, we can divide this volume of $\mathrm{CO}_{2}$ to be released by the average volume of a typical bubble of $0,5 \mathrm{~mm}$ in diameter. A huge number close to $10^{8}$ is found! Actually, the so-called effervescence process, which enlivens champagne and sparkling wines tasting, is the result of the fine interplay between $\mathrm{CO}_{2}$ dissolved gas molecules, tiny air pockets trapped within microscopic particles during the pouring process, and some both glass and liquid properties. Bubbles nucleated on the glass wall rise toward the champagne surface, where tens of thousands of them finally collapse during champagne tasting.

In this tutorial review, a collection of collapsing-bubble-driven phenomena found at the top of a glass poured with champagne are gathered and characterized, through high-speed photography and high-speed imaging. This review illustrates how the collapse of bubbles at the liquid surface undoubtedly constitutes the most intriguing, functional, and visually appealing step of champagne bubbles' fleeting life.

\section{Shape of floating bubbles before collapse.}

Champagne bubbles, that travel approximately $10 \mathrm{~cm}$ between their nucleation sites and the liquid surface, reach the free surface with a radius $R$ of order of $0.5 \mathrm{~mm}$. At the free surface, the shape of a bubble results from a balance between two opposing effects: the buoyancy $F_{B}$, of order of $\rho g \pi R^{3}$, which tends to make it emerge from the free surface and the capillary force $F_{C}$ inside the hemispherical thin liquid film, of order of $(\gamma / R) \pi R^{2}=\gamma \pi R$, which tends to maintain the bubble below the surface. $(\gamma / R)$ is the excess of pressure, due to Laplace law, inside the curved thin liquid film of the bubble-cap, and $\pi r^{2}$ is the order of magnitude of the emerged bubble-cap's area. Comparing these two opposing forces is equivalent to compare the bubble radius, $R \approx 0.5 \mathrm{~mm}$, with the capillary length of the liquid medium, 
$\kappa^{-1}=\sqrt{\gamma / \rho g} \approx 2 \mathrm{~mm}$, (where $\gamma, \rho$ and $g$ are respectively the liquid surface tension, the liquid density and the acceleration due to gravity). Champagne bubbles' radii being significantly smaller than the capillary length, gravity will be neglected in front of capillary effects. Consequently, like a tiny iceberg, a floating bubble only slightly emerges from the liquid surface, with most of its volume remaining below the free surface. The scheme displayed in Figure 1 presents the various geometrical parameters linked with bubbles trapped near the free surface in a bubble raft composed of millimetric bubbles (as are champagne bubbles). The following reasoning does not take into account interactions between adjoining bubbles (no dimple effects, for example).

\section{Figure 1}

Let us denote, $R_{1}$ and $R_{2}$, the radii of curvature of the emerged bubble-cap and the immersed bubble volume, respectively. Therefore, since the thin emerging bubble-cap possesses two interfaces whereas the immerged part of the bubble possesses only one, the excess of pressure inside a bubble, $\Delta P$, may be written as follows (according to the Laplace law):

$$
\Delta P=\frac{4 \gamma}{R_{1}}=\frac{2 \gamma}{R_{2}} \Rightarrow R_{1}=2 R_{2}
$$

Consequently, the radius of curvature of the emerged bubble-cap is approximately twice that of the submerged bubble volume. Furthermore, since most of the bubble volume remains below the liquid surface, $R_{2} \approx R$ and consequently, $R_{1} \approx 2 R$. Obviously, the transition between these two zones of different curvature does not appear so abruptly. A complete numerical study of the shape taken by a bubble trapped at a gas/liquid interface was 
conducted by Laurent Duchemin, in the limit of small and large bubble radii. ${ }^{10,11}$ The radius of the emerged bubble-cap, $r$, as shown in Figure 1, can also be estimated by equaling the bubble buoyancy with the capillary pressure inside the thin film as:

$$
\rho g\left(\frac{4}{3} \pi R^{3}\right) \approx \frac{\gamma}{R} \pi r^{2} \Rightarrow r \approx \sqrt{\frac{4}{3}} \frac{R^{2}}{\kappa^{-1}} \approx 0.14 \mathrm{~mm}
$$

Finally, the order of magnitude of the angle between the z-axis and the end of the bubble-cap may also be easily determined as, $\psi \approx \sin ^{-1}(r / 2 R) \approx 8^{\circ}$.

\section{Dynamics of a bubble-cap's aperture.}

At the free surface, since bubbles' radii are significantly smaller than the capillary length, the liquid films of bubble-caps progressively get thinner due to capillary drainage. When the liquid film of a bubble-cap reaches a critical thickness, it becomes fragile and finally ruptures. Since the pioneering work of Lord Rayleigh, in the late $19^{\text {th }}$ century, ${ }^{12}$ the rupture of thin films has been widely experimentally, theoretically and numerically investigated. It was found that a hole appears in the film, surrounded by a rim which collects the liquid and that propagates very quickly driven by surface tension forces. Balancing inertia with surface tension, Culick proposed the following velocity for the growing hole in a thin rupturing liquid sheet, ${ }^{13}$

$$
u_{\text {Culick }} \approx \sqrt{\frac{2 \gamma}{\rho e}}
$$

where $e$ is the thickness of the liquid film. The latter expression has already been experimentally confirmed numerous times for the bursting of thin liquid films of low 
viscosity (see for example the pioneering work by McEntee and Mysels, ${ }^{14}$ and also that by Pandit and Davidson, ${ }^{15}$ where beautiful snapshots of the disintegration of soap bubbles are presented).

\section{Figure 2}

In the case of a spherical cap with a radius of curvature $2 R$, rupturing from its axis of symmetry (the z-axis) as schematized in Figure 2, the velocity $u$ of the propagating liquid rim (of mass $M$ ) is ruled by the following equation:

$$
\frac{d}{d t}(M u)=2 \gamma(2 R) \sin \theta d \varphi
$$

By replacing in the latter equation, the mass of the liquid rim by its expression, $M=\rho e(2 R)^{2}(1-\cos \theta) d \varphi$, the velocity $u$ by $2 R(d \theta / d t)$ and by developing, one obtains the following differential equation:

$$
\rho e(2 R)^{2}\left[\frac{d^{2} \theta}{d t^{2}}(1-\cos \theta)+\sin \theta\left(\frac{d \theta}{d t}\right)^{2}\right]=2 \gamma \sin \theta
$$

Finally, by considering only the constant velocity solution $\left(d^{2} \theta / d t^{2}=0\right)$, one obtains the following expression $u$ for the growing hole,

$$
u=2 R \frac{d \theta}{d t}=\sqrt{\frac{2 \gamma}{\rho e}}=u_{\text {Culick }}
$$


which is the same as that derived by Culick. The thickness $e$ of a millimetric champagne bubble-cap was already experimentally determined by the classical micro-interferometric technique and found to be of order of $10^{-6}$ to $10^{-7} \mathrm{~m} .{ }^{16}$ As a result, by replacing in equation (74) the known values of $\gamma, \rho\left(\approx 10^{3} \mathrm{~kg} \mathrm{~m}^{-3}\right)$ and $e, u$ is expected to be of order of $10 \mathrm{~m} \mathrm{~s}^{-1}$ for a rupturing champagne liquid film. Finally, the characteristic time scale $\tau$ of a millimetric bubble-cap's disintegration should be around:

$\tau \approx r / u \approx 1.4 \cdot 10^{-4} / 10 \approx 10 \mu \mathrm{s}$

\section{An Estimation of the shear stresses during a bubble-cap's aperture.}

To estimate the shear stress during the bubble-cap's disintegration, we will use an alternative approach, inspired by Clarkson et al, based on the energy dissipation rate. ${ }^{17}$ The energy dissipation rate $\varepsilon$ is the rate at which energy is dissipated per unit mass of liquid,

$\varepsilon=\frac{1}{m} \frac{d E_{\mathrm{D}}}{d t}$

where $m$ is the mass of the liquid film $\left(m \approx \rho e \pi r^{2}\right)$. Half of the total surface free energy $E_{\mathrm{T}}$ of the bubble-cap (where $E_{\mathrm{T}}=2 \gamma \pi r^{2}$, due to the both gas/liquid interfaces) is dissipated by viscous effects into the liquid rim around the growing hole, the other half being converted into kinetic energy. ${ }^{13-15}$ Therefore, $E_{\mathrm{D}} \approx E_{\mathrm{T}} / 2$. As a result, during the bubble-cap's disintegration phase, the energy dissipation rate can be approximated as:

$\varepsilon \approx\left(\frac{1}{\rho e \pi r^{2}}\right) \frac{\gamma \pi r^{2}}{\tau} \approx \frac{\gamma}{e \rho \tau}$ 
As presented in the work by Clarkson et al ${ }^{17}$ we used the turbulence theory to estimate the shear stress $\sigma$ in the liquid rim, from the energy dissipation rate by use of the following relationship, ${ }^{18}$

$$
\sigma \approx \eta\left(\frac{\varepsilon}{v}\right)^{1 / 2}
$$

where $v$ is the kinematic viscosity defined as, $v=\eta / \rho$. Finally, combining equations (8) and (9) yields the following expression for the shear stress encountered as the emerging bubblecap disintegrates:

$$
\sigma \approx\left(\frac{\gamma \eta}{e \tau}\right)^{1 / 2}
$$

By replacing in equation (10) the known values of $\gamma, \eta, e$ and $\tau$ in the present situation, shear stresses in the propagating liquid rim are expected to be comprised in between $3 \times 10^{3} \mathrm{~N} \mathrm{~m}^{-2}$ and $9 \times 10^{3} \mathrm{~N} \mathrm{~m}^{-2}$, depending on the film thickness as it ruptures.

\section{The bursting process as captured through high-speed imaging}

It is now generally recognized that bubbles bursting at a liquid surface eject two kinds of droplets: ${ }^{19}$ (i) small droplets called film drops, formed as the film of the emerged bubble-cap disintegrates, and (ii) droplets formed by the collapse of the bottom of the bubble, called jet drops (see Figure 3). Nevertheless, it was shown that bubbles with a diameter less than about $2 \mathrm{~mm}$ produce no film drops as they burst. ${ }^{19}$ Because the champagne bubbles' diameter rarely 
exceeds about $1 \mathrm{~mm}$ as they reach the liquid surface, it can be concluded that only jet drops constitute the cloud of droplets above the liquid surface.

\section{Figure 3}

Following equation (6), the characteristic time scale needed for the disintegration of the bubble-cap of millimetric bubbles is of order of $10 \mu \mathrm{s}$. During this extremely brief initial phase, the bulk shape of the bubble is literally "frozen", and a nearly millimetric open cavity remains as a tiny indentation in the liquid surface (see for example the two high speed photographs displayed in Figure 4). ${ }^{20}$

\section{Figure 4}

Then, a complex hydrodynamic process ensues, causing the collapse of the submerged part of the bubble and projecting into the air a liquid jet (the often so-called Worthington jet ${ }^{21}$ ) which quickly breaks up into tiny droplets of liquid (called jet drops). This process is indeed characteristic of every carbonated beverage. Generally speaking, the number, size, and velocity of jet drops produced during bubble collapse depend on both the size of the initial bursting bubble and some liquid properties (such as its surface tension, density and viscosity). ${ }^{22-25}$ In Figure 5, a reconstructed time-sequence frozen through high-speed photography is displayed. It shows the formation of a tiny liquid jet, caused by the collapse of a champagne bubble, and which breaks up into several droplets. ${ }^{20}$

\section{Figure 5}




\section{Close-up on the Worthington jet}

The so-called Rayleigh-Plateau instability is a capillary wave, which quickly develops along the Worthington jet, and which is responsible for its breakup into several droplets. The photograph displayed in Figure 6a shows the split-second traveling of the Rayleigh-Plateau instability which develops along the jet, as frozen through high-speed photography. It is compared with the Rayleigh-Plateau instability which develops along a cylindrical water stream falling from a leaking faucet (see Figure $6 \mathbf{b}$ ).

\section{Figure 6}

The length scale $l$ of the upward jet radius being of order of $100 \mu \mathrm{m}$, its corresponding Bond number is therefore expected to be $B o=\left(\rho g l^{2}\right) / \gamma \approx 2 \cdot 10^{-3}<<1$. Moreover, in case of millimetric bubbles, the upward jet velocity $U$ being of order of $5 \mathrm{~m} / \mathrm{s},{ }^{10,11}$ the Reynolds number of the jet is therefore of order of $\operatorname{Re}=(\rho U l) / \eta \approx 10^{2}>>1$. Therefore, the RayleighPlateau instability, which develops along the champagne jet, results from a balance between inertia $\left(\rho d U / d t \approx \rho U / \tau_{\mathrm{R}}\right)$ and surface tension gradients $\left(\nabla P \approx \gamma / l^{2}\right)$, with $\tau_{\mathrm{R}}$ being the characteristic time scale for the instability to develop. Finally, by equating the two latter expressions, the characteristic time scale $\tau_{\mathrm{R}}$ for the Rayleigh-Plateau instability to develop in the jet is of order of:

$$
\tau_{\mathrm{R}} \approx \frac{\rho U l^{2}}{\gamma} \approx \frac{10^{3} \times 5 \times\left(10^{-4}\right)^{2}}{5 \times 10^{-2}} \approx 10^{-3} \mathrm{~s} \approx 1 \mathrm{~ms}
$$

Figure 7 
The collapse of single millimetric bubbles was also filmed through high-speed video camera.

A typical time sequence is displayed in Figure 7. The Rayleigh-Plateau instability seems to develop from frame 6 . It is worth noting that the characteristic time of about $1 \mathrm{~ms}$ needed to develop the Rayleigh-Plateau instability on the tiny Worthington jet is totally self-consistent with the one theoretically determined following equation (11).

In comparison, the characteristic time scale required for the Rayleigh-Plateau instability to develop in a millimeter-size water stream falling from a leaking faucet is rather of order of 3 ms. $^{26}$

\section{Bouncing and in-flight coalescence of champagne droplets}

The most complex phenomena of fluid dynamics are at play on top of a champagne flute. This is illustrated for example in Figure $\mathbf{8}$ with the life of two champagne droplets (previously ejected by bursting bubbles), almost dancing together in close vicinity.

\section{Figure 8}

A close examination of the largest droplet reveals that it rebounds on the champagne surface. This surprising phenomenon is made possible by the thin layer of air underneath the droplet. As the droplet approaches the free surface, the thin air layer pressurizes due to lubrication effects. This pressure rise makes it possible for the evanescent air to repel the dense droplet. ${ }^{27}$ But while rebounding, the larger droplet hits the smaller one. Due to its higher pressure, the smaller droplet drains into the larger, on the rapid capillary timescale $\left(\tau \approx \sqrt{\rho R^{3} / \gamma} \approx 0.1 \mathrm{~ms}\right)$. The resulting strongly deformed merger then vibrates over a slightly slower capillary timescale (now, the radius of the final drop is slightly bigger). This final droplet still rebounds one, two and three times before eventually coalescing with the champagne bath. ${ }^{28-29}$ 


\section{A paternoster for surface active molecules}

As champagne or sparkling wine is poured into a glass, the myriad of ascending bubbles collapse and therefore radiate a multitude of tiny droplets above the free surface, into the form of very characteristic and refreshing aerosols, as shown in the photograph displayed in Figures 9. Those tiny droplets, ejected up to several centimeters above the liquid surface, partly evaporate themselves, thus accelerating the transfer of the numerous aromatic volatile organic compounds above the liquid surface. This very characteristic fizz considerably enhances the flavor release in comparison with that from a flat wine for example. Laser tomography techniques were applied to freeze the huge number of bursting events and the myriad of droplets ejected above champagne glasses in real consuming conditions (see the tomography of the droplets' cloud above the surface of a coupe displayed in Figure 10). ${ }^{6}$

\section{Figure 9}

Figure 10

On a larger scale indeed, sea spray, which transports dissolved gases, salts, and biological materials to the atmosphere (and therefore influences climate), is largely attributed to aerosols produced by an estimated $10^{18}$ to $10^{20}$ bubbles that rupture every second across the oceans. ${ }^{30-}$

${ }^{32}$ Marine aerosols account for the majority of the global natural aerosol flux and consequently may have a significant impact on the Earth's radiative balance and biogeochemical cycling. ${ }^{33 \text {, }}$ ${ }^{34}$ On a smaller scale, it is worth noting that bubble-bursting-driven aerosols have been implicated in the transmission of diseases in swimming pools and hot tubs. ${ }^{35}$

It is indeed well known that preferential adsorption of surfactants at the air-solution interface occurs as a result of the amphiphilic properties of surfactants, with the water-soluble moiety 
plunging into the solution and the hydrophobic component in contact with the air. Enrichment of surfactant materials - in the sea-surface microlayer, and in atmospheric aerosols - has long been well characterized. ${ }^{36-40}$ Adsorption of surfactants is even considerably increased at the sea surface during rough sea conditions, when wave breaking action causes air bubbles to be trapped under the water surface. ${ }^{34}$ Actually, bubbles trapped in the liquid bulk considerably increase exchange surfaces between the sea bulk and the atmosphere. Bubbles drag surfactants along their way through the liquid bulk, reach the sea surface, to finally burst and eject aerosol droplets into the atmosphere. Air bubbles trapped during rough sea conditions were found to increase surfactant concentrations in aerosols by several orders of magnitude compared with those found in the liquid bulk. ${ }^{34}$ Recently, Bird et al proved that, by rupturing, a single large bubble (with several centimeters in diameter) can fold and entrap air as it retracts, thus leading to the creation of a ring of small daughter millimetric bubbles which burst in turn. ${ }^{41}$ This phenomenon is believed to considerably increase the number and efficiency of aerosol dispersal.

From a conceptual point of view, the situation found in glasses poured with champagne or sparkling wine is finally very similar to that described above. Champagne holds indeed hundreds of surface active compounds. Once champagne is poured into a glass, bubbles nucleated on the glass wall drag champagne surfactants along their way through the liquid bulk. ${ }^{6}$ Surfactants dragged along with ascending bubbles finally reach the free surface and concentrate themselves at the air/champagne interface. At the free surface of a glass poured with champagne, the ever-increasing concentration of surfactants was indeed indirectly evidenced by observing the ever-increasing lifetime of bubbles with time. ${ }^{8}$ Actually, the everincreasing surface concentration of surfactants progressively changes the boundary condition on the bubble surface from slip to non-slip, thus reducing the drainage velocity and extending the bubble's lifetime. Quite recently, the formation of adsorption layers of amphiphile 
macromolecules at the air/champagne interface were directly evidenced through ellipsometry and Brewster angle microscopy (BAM) experiments. ${ }^{42,43}$

Based on a phenomenological analogy between the fizz of the ocean and the fizz in Champagne wines, it was hypothesized a few years ago, that aerosols found in the headspace above a glass poured with champagne could considerably enhance the fragrance release of champagne by bringing chemical compounds to the taster's nostrils, showing both surface activity and organoleptic interest. ${ }^{20}$ Recently, ultrahigh resolution mass spectrometry (ICRFT/MS) was used in order to analyze the aerosol released by champagne bubbles. ${ }^{44}$ Champagne droplets following bubble collapse were definitely found to be more concentrated with various surface active compounds - some of them showing indeed organoleptic interest than the champagne bulk (see Figure 11). Each dot displayed in Figure 11 represents the concentration factor of a given compound found in the aerosols (i.e., the ratio of its concentration in the aerosols to its concentration in the bulk, below the champagne surface). These compounds, mostly including saturated and unsaturated fatty acids, act as surfactants (i.e., as double-ended compounds with one end attracted to the liquid phase and another that shuns it). It was suggested that champagne bubbles drain these compounds out of the liquid bulk toward the liquid surface, with the hydrophobic end attracted by the bubble's airy inside and the hydrophilic end attracted by the liquid outside. The bubbles then rise to the surface of the glass where they pop, releasing the compounds as aerosols. This recent discover supports the idea that rising and collapsing bubbles act as a continuous paternoster lift for aromas in every glass of champagne. Aerosols were thus found to hold the organoleptic "essence" of champagne.

\section{Figure 11}




\section{Capillary-driven flower-shaped structure around bubbles collapsing in a monolayer}

The close observation of bubbles collapsing at the free surface of a glass poured with champagne also revealed another unexpected and lovely phenomenon. A few seconds after pouring, and after the collapse of the foamy head, the surface of a champagne flute is covered with a layer of quite monodisperse bubbles - a kind of bubble raft, or 2D foam, where each bubble is generally surrounded by six neighbouring bubbles (see Figure 12). Bubbles arrange themselves in an approximate hexagonal pattern.

\section{Figure 12}

While snapping pictures of the bubble raft after pouring, pictures of bubbles collapsing close to one another were taken. When the bubble-cap of a bubble ruptures and leaves an open cavity at the free surface, adjacent bubble-caps are sucked towards this empty cavity and create unexpected and short-lived flower-shaped structures, unfortunately invisible to the naked-eye (see for example the high speed photographs displayed in Figure 13). ${ }^{45}, 46$ Shear stresses induced by bubbles trapped in the close vicinity of a collapsing one are even better visualized on the high-speed photograph displayed in Figure 14, where the bubble raft is not complete. Such a behaviour first appeared counter-intuitive. Paradoxically, adjacent bubblecaps are sucked and not blown-up by bursting bubbles, contrary to what could have been expected at first glance.

Figure 13

Figure 14 
Actually, after the disintegration of a bubble-cap, the hexagonal symmetry around adjoining bubbles is suddenly locally broken. Therefore, the symmetry in the field of capillary pressure around adjoining bubbles is also locally broken. Capillary pressure gradients all around the now empty cavity are detailed in Figure 15.

\section{Figure 15}

Signs $+/$ - indicate a pressure above/below the atmospheric pressure $P_{0}$. Finally, inertia and gravity being neglected, the full Navier-Stokes equation applied to the fluid within the thin liquid film of adjoining bubble-caps drawn by capillary pressure gradients, reduces itself to a simple balance between the capillary pressure gradients and the viscous dissipation as follows, ${ }^{46}$

$$
\eta(\Delta \vec{u})_{S}=(\vec{\nabla} P)_{S}
$$

where $u$ is the velocity in the thin liquid film of adjacent bubble-caps, $\eta$ is the champagne viscosity, $\vec{\nabla} P$ are the capillary pressure gradients, and $s$ being the axial coordinate which follows the bubble-cap's curvature and along which the fluid within the thin film is displaced. The asymmetry in the capillary pressure gradients distribution around a bubble-cap adjacent to an empty cavity is supposed to be the main driving force of the violent sucking process experienced by a bubble-cap in touch with a bursting bubble. Actually, due to higher capillary pressure gradients, the liquid flows that develop in the half part of the bubble-cap close to the open cavity are thus expected to be higher than those which develop in the rest of the bubblecap. It ensues a violent stretching of adjoining bubble-caps toward the now empty cavity, which is clearly visible on the photographs displayed in Figures 13 and 14. 
More recently, those flower-shaped structures have been observed during the coarsening of bidimensional aqueous foams, obtained by mixing a surfactant, sodium dodecyl sulphate (SDS), with pure water. ${ }^{47}$ But it is worth noting that this lovely and short-lived process was first observed at the top of a champagne flute.

During this sudden stretching process, adjacent bubble-caps areas significantly increase. A systematic image analysis of numerous time sequences similar to that displayed in Figure 5 demonstrated an average increase $\Delta A$ around $15 \%$, of bubble-caps areas adjacent to a central collapsing bubble. The free energy of such a system will be supposed to be mainly stored as surface free energy. Consequently, the density of free energy per unit of volume in the liquid film of a distorted bubble-cap (one petal of the flower-shaped structure) can be evaluated as follows, ${ }^{45}$

$\left(\frac{\Delta E}{V}\right)_{\text {bubble-cap }} \approx \frac{2 \gamma \Delta A}{A e} \approx \frac{0.15 \times 2 \gamma}{e} \approx 10^{4}-10^{5} \mathrm{~J} \mathrm{~m}^{-3}=10^{4}-10^{5} \mathrm{~N} \mathrm{~m}^{-2}$

where $\Delta E$ is the corresponding surface free energy in excess during the stretching process, $V$ is the volume of the thin liquid film of the emerging bubble-cap, $A$ is the emerging bubble-cap area, and $e$ is the thickness of the thin liquid film of the bubble-cap (of order of $10^{-6}-10^{-7} \mathrm{~m}$ ). Therefore, the liquid flows induced by the capillary pressure gradients are responsible for a density of energy per unit of volume dimensionally equivalent to shear stresses of order of $10^{4}-10^{5} \mathrm{~N} \mathrm{~m}^{-2}$ (depending on the film thickness). By comparison, in previous studies, numerical models conducted to stresses of order of (only) $10^{3} \mathrm{~N} \mathrm{~m}^{-2}$ in the boundary layer around single millimetric collapsing bubbles. ${ }^{10,48}$ Therefore, stresses in the bubble-caps of bubbles adjacent to collapsing cavities appear to be, at least, one order of magnitude higher than those observed around single collapsing cavities, and also even higher than those 
developed by viscous dissipation as a bubble-cap disintegrates. Intuitively, this is definitely not so surprising. Actually, after a bubble-cap's aperture, the now empty cavity has to collapse to recover the horizontality of the liquid surface. The potential energy of such an unstable situation is the sum of two contributions: first, the gravitational energy associated with the buoyancy of the open cavity (of order of $\rho g \pi R^{4}$ ) and second, the difference of surface free energy between an hemispherical open cavity of radius $R$ and a flat circular surface of radius $R$ (of order of $\gamma \pi R^{2}$ ). The ratio of the gravitational energy to the surface free energy is called the Bond number, $B o=\left(\rho g R^{2}\right) / \gamma$. In the case of a $1 \mathrm{~mm}$ diameter bubble, $B o$ being of order of $5 \cdot 10^{-2}$, the main source of energy is undoubtedly the surface free energy. Now, it should be noted that the same driving potential energy is responsible for the single cavity collapse and for the collapse of the cavity surrounded by neighboring bubble-caps. But, the volume of liquid displaced in the thin film of adjoining bubble-caps being much less than that of the boundary layer drawn, it logically induces higher energy dissipation rates per unit of volume and therefore higher strains in the "petals" of the flower-shaped structure. Finally, while absorbing the energy released during a bubble collapse, as so many tiny air-bags would do, adjoining bubble-caps store this energy into the thin liquid film of emerging bubble-caps, leading finally to stresses much higher than those observed in the boundary layer around single millimetric collapsing bubbles.

In addition to purely physicochemical reasons, biological reasons are also readily found for the investigation of such flower-shaped structures around bubbles collapsing in a bubble raft. Actually, in the biological industry, animal cells cultivated in bio-reactors were shown to be seriously damaged or even killed by the bursting of gas bubbles used to aerate the culture medium. ${ }^{49,50}$ It has even been suggested that structural deformations of adjacent tissues are induced by bubble collapse during laser-induced angioplasty. ${ }^{51,52}$ Kunas and Papoutsakis evaluated the critical shear stresses needed to cause irreversible damages to animal cells. ${ }^{50}$ 
They found critical lethal stresses in the range between $10^{3} \mathrm{~N} \mathrm{~m}^{-2}$ and $10^{4} \mathrm{~N} \mathrm{~m}^{-2}$. Therefore, by developing stresses of order of $10^{4}-10^{5} \mathrm{~N} \mathrm{~m}^{-2}$, bubbles bursting in a bubble raft should be potentially even more dangerous for micro-organisms or biological tissues trapped in the thin film of these "fast-stretched bubble-caps".

\section{Avalanches of bursting events in the bubble raft?}

Actually, avalanches of popping bubbles were put in evidence during the coarsening of bidimensional and three dimensional aqueous foams. ${ }^{47,53}$ How does the bubble raft behave at the surface of a flute poured with champagne? Does a bursting bubble produce a perturbation which extends to the neighboring bubbles and induce avalanches of bursting events which finally destroy the whole bubble raft? In case of champagne wines, a few time sequences of bubbles bursting in the bubble raft have been captured with a high-speed video camera. One of them is displayed in Figure 16.

\section{Figure 16}

Between frame 1 and frame 2, the bubble indicated with the black arrow has disappeared. In frame 2, neighboring bubbles are literally sucked toward this now bubble-free area. Then, neighboring bubbles oscillate during a few milliseconds and progressively recover their initial hemispherical shape. In conclusion, in the case of bubbles adjacent to collapsing ones, despite high shear stresses produced by a violent sucking process, bubbles adjacent to collapsing ones were never found to rupture and collapse in turn, thus causing a chain reaction. At the free surface of a flute poured with champagne, bursting events appear to be spatially and temporally non correlated. The absence of avalanches of bursting events seems to be linked to the champagne viscosity (which is about $50 \%$ higher than that of pure water). ${ }^{47}$ 
It can also be noted that a tiny daughter bubble, approximately ten times smaller than the initial central bubble, has been entrapped during the collapsing process of the central cavity (as clearly seen in frames 3 and 4 of Figure 16). Bubble entrapment during the collapsing process was already experimentally and numerically observed with single millimetric collapsing bubbles, ${ }^{10,54}$ including champagne bubbles. ${ }^{20}$ This bubble entrapment process can also be observed as drops impact on liquid surfaces. ${ }^{55-59}$

\section{And what about the Worthington jet?}

Since we are dealing with bubbles collapsing at a free liquid/gas interface, we are logically tempted to wonder about the dynamics of the famous Worthington jet. Does it exist, as in the single collapsing bubble case, or does the roughly hexagonal neighboring bubble pattern around a collapsing cavity strongly modify and even prevent its formation?

Following equation (11), changes in the upward jet velocity $U$ and/or changes in its radius $l$, in comparison with the single bubble collapse case, could finally strongly affect the overall dynamics of the Rayleigh-Plateau instability which breaks the jet into droplets.

Once more, intuitively, differences between the dynamics of the Worthington jet could be expected. Actually, the bulk shape of bubbles adjacent to the empty cavity left by the central collapsing bubble strongly changes the geometry of the system beneath the free surface. Such a situation could therefore modify the converging liquid flows all around the empty cavity, thus probably modifying in turn the overall dynamics of the upward liquid jet. Actually, a few snapshots captured the formation of a Worthington jet for collapsing bubbles being surrounded by the hexagonal pattern of six neighboring bubbles. Figure 17 compares a liquid jet issued from a bubble collapsing in a bubble raft with a liquid jet issued from the collapse of a single champagne bubble. ${ }^{46}$ 


\section{Figure 17}

The jet following the single collapsing bubble seems to be better "developed" than that following the bubble collapsing in touch with neighboring bubbles. This seems to confirm that the radial flow around the cavity is sufficiently affected by the surrounded bubbles to weaken the resulting jet. The same trend has been noticed on the few snapshots that froze the Worthington jet in a bubble raft, as well as on several time sequences taken through the highspeed video camera (see Figure 18 for example).

\section{Figure 18}

\section{A particular situation: as the jet deviates from vertical}

Liquid jets shooting out from single collapsing bubbles are pictured as perfectly vertical. But sometimes, largely tilted jets are produced. One of such rare event has been captured in the sequence reported Figure 19.

\section{Figure 19}

The Worthington jet following bubble collapse clearly deviates from vertical. How horizontal momentum is gained in these jets is still an unsettled question. As a tentative explanation, it can be noted that those jets typically originate from bubbles located at the edge of a bubble raft. It has already been pointed out earlier that raft bubbles or isolated bubbles do not give rise to the same jet. The reason for this difference lies in the initial shape of the collapsing bubble. When isolated, the bubble is completely axisymmetric and so are the capillary waves 
riding on the bubble surface. The resulting jet preserves this axisymmetry and is vertical. When embedded in a raft, the bubble wall is no longer axisymmetric. Rather, it is now faceted with a hexagonal symmetry. But this local loss of azimuthal symmetry is of no consequence for the developing jet. Now when the bubble is located on the edge of the raft, there is a complete loss of symmetry in the initial shape. This strong asymmetry reflects in turn in the developing capillary waves, and eventually in the resulting tilted jet. Such asymmetry-induced momentum is typical of, e.g. strong liquid jets produced during the collapse of cavitation bubble cloud and responsible for cavitation damage. ${ }^{60}$

\section{Figure 20}

The high-speed photograph displayed in Figure 20 froze the champagne liquid jet in a quite particular situation. ${ }^{61}$ On the right side of this picture, the collapsing bubble is bordered by three neighboring bubbles, whereas on the left side, there are no adjoining bubbles. The hexagonal symmetry is broken. In this case, the tiny liquid jet (perfectly vertically oriented in case of a single collapsing bubble, as in Figure 5) seems to deviate from vertical. The jet is tilted toward the "bubble-free" area, as in the sequence displayed in Figure 19. There are most probably no enological consequences of such a situation, but experts in the science of bubbles and foams ask themselves why such a deviation from vertical is observed.

Another example of the relevance of dynamical phenomena in the bursting of champagne bubbles and high-speed liquid jets development is illustrated Figure 21.

Figure 21 
Right after the bubble rupture, a capillary wave is emitted and rides radially outwards. As the wave reaches distant bubbles, their synchronous explosion is observed, as if the wave had triggered the bubble rupture. This is yet another example of the importance of dynamics in the behaviour of bubbles in real configurations. As a last demonstration of the wave-induced nature of bubble rupture, a careful examination of Figure 21 shows that the Worthington jets resulting from the bubbles collapse are tilted towards the initial collapsing bubble. It seems to be a clear connection between wave-induced rupture and jet tilting. This wave-induced horizontal momentum source is another form of asymmetry, and is reminiscent of the forward source of momentum of « walking droplets », rebounding on a vibrating liquid bath. ${ }^{27}$

\section{Conclusion}

In this article, a non exhaustive review of collapsing-bubble-driven phenomena found at the top of a glass poured with champagne were gathered and characterized, through high-speed photography and high-speed imaging. After an overview on the bubble collapse process per $s e$, the resulting Worthington jet has been described along with a collection of phenomena which can play a role in the droplets production, most of them being still under investigation. Moreover, due to the high complexity of such collective phenomena, further experimental observations, combined with numerical simulations, are soon to be conducted in order to better understand the role of neighboring bubbles on the dynamics of the jet formation and its breakup into droplets. The world's most famous sparkling wine is still far from unveiling all the secrets hidden behind its fizzy sensation. 


\section{Literature cited}

(1) D. Lohse, Phys. Today 56, 36 (2003)

(2) S. Sirsi, M. Borden, Bubble Science, Engineering and Technology 1, 3 (2009)

(3) N. Rapoport, A. Efros, D. Christensen, A. Kennedy, K.-H. Nam, Bubble Science, Engineering and Technology 1, 31 (2009)

(4) P. Kogan, R. Gessner, P. Dayton, Bubble Science, Engineering and Technology 2, 3 (2010)

(5) A. Peart, J. R. Evans, Bubble Science, Engineering and Technology 3, 64 (2011)

(6) G. Liger-Belair, G. Polidori G, P. Jeandet, Chem. Soc. Rev. 37, 2490 (2008)

(7) G. Liger-Belair, Uncorked : the science of champagne (Princeton University Press, Princeton, 2004)

(8) G. Liger-Belair, Ann. Phys. (Paris) 31, 1 (2006)

(9) G. Liger-Belair, G. Polidori G, V. Zéninari, Anal. Chim. Acta, in press (2012)

(10) L. Duchemin, Ph.D. thesis, Université d'Aix-Marseille II, (2001)

(11) L. Duchemin, S. Popinet, C. Josserand, S. Zaleski, Phys. Fluids 14, 3000 (2002)

(12) Lord Rayleigh, Proc. R. Inst. 13, 261 (1891): Reproduced in Scientific Papers, Vol. 3 (Dover, New-York, 1964)

(13) F.E. Culick, J. Appl. Phys. 31, 1128 (1960)

(14) W.R. McEntee, K.J. Mysels, J. Phys. Chem. 73, 3018 (1969)

(15) A.B. Pandit, J.F. Davidson, J. Fluid Mech. 212, 11 (1990)

(16) J. Senée, B. Robillard, M. Vignes-Adler, Food Hydrocolloids 13, 15 (1999)

(17) J.R. Clarkson, Z.F. Cui, R.C. Darton, J. Colloid Interface Sci. 215, 323 (1999)

(18) H. Schlichting, Boundary Layer Theory (McGraw-Hill, New York, 1979)

(19) F.J. Resch, J.S. Darrozes, G.M. Afeti J. Geophys. Res. 91, 1019 (1986) 
(20) G. Liger-Belair, H. Lemaresquier, B. Robillard, B. Duteurtre, P. Jeandet, Am. J. Enol. Vitic. 52, 88 (2001)

(21) A.M. Worthington, A Study of Splashes (Macmillan, New York, 1963)

(22) D.E. Spiel, J. Geophys. Res. 99, 10289 (1994)

(23) D.E. Spiel, J. Geophys. Res. 100, 4995 (1995)

(24) D.E. Spiel, J. Geophys. Res. 102, 5815 (1997)

(25) D.E. Spiel, J. Geophys. Res. 103, 24907 (1998)

(26) P.G. De Gennes, F. Brochard-Wyart, D. Quéré, Capillarity and Wetting Phenomena (Springer-Verlag, New York, 2004)

(27) Y. Couder, S. Protiere, E. Fort, A. Boudaoud, Nature 437, 208 (2005)

(28) S.T. Thoroddsen, K. Takehara, Phys. Fluids 12, 1265 (2000)

(29) F. Blanchette, T.P. Bigioni, Nat. Phys, 2, 254 (2006)

(30) A.H. Woodcock, C.F. Kientzler, A.B. Arons, D.C. Blanchard, Nature 172, 1144 (1953)

(31) F. MacIntyre, J. Geophys. Res. 77, 5211 (1972)

(32) J. Wu, Science 212, 324 (1981)

(33) C.D. O’Dowd, M.C. Facchini, F. Cavalli, D. Ceburnis, M. Mircea, S. Decesari, S. Fuzzi, Y.J. Yoon, J.-P. Putaud, Nature 431, 676 (2004)

(34) C.D. O’Dowd, G. de Leeuw, Philos. Trans. R. Soc. A 365, 1753 (2007)

(35) L.T. Angenent, S.T. Kelly, A. St Amand, N.R. Pace, M.T. Hernandez, Proc. Natl. Acad. Sci. USA 102, 4860 (2005)

(36) W.R. Barger, W.D. Garret, J. Geophys. Res. 75, 4561 (1970)

(37) D.C. Blanchard, Tellus B 42, 200 (1990)

(38) R.S. Tseng, J.T. Viechnicki, R.A. Skop, J.W. Brown, J. Geophys. Res. 97, 5201 (1992) 
(39) C. Oppo, S. Bellandi, N. Innocenti, A. Stortini, G. Loglio, E. Schiavuta, R. Cini, Mar. Chem. 63, 235 (1999)

(40) S. Ekström, B. Nozière, M. Hultberg, T. Alsberg, J. Magnér, E.D. Nilsson, P. Artaxo, Biogeosciences 7, 387 (2010)

(41) J.C. Bird, R. de Ruiter, L. Courbin, H.A. Stone, Nature 465, 759 (2010)

(42) N. Péron, A. Cagna, M. Valade, C. Bliard, V. Aguié-Béghin, R. Douillard, Langmuir 17, $791(2001)$

(43) N. Péron, J. Meunier, A. Cagna, M. Valade, R. Douillard, J. Microscopy 214, 89 (2004)

(44) G. Liger-Belair, C. Cilindre, R. Gougeon, M. Lucio, I. Gebefügi, P. Jeandet, P. Schmitt-Kopplin, Proc. Natl. Acad. Sci. USA 106, 16545 (2009)

(45) G. Liger-Belair, B. Robillard, M. Vignes-Adler, P. Jeandet, C. R. Phys. 2, 775 (2001)

(46) G. Liger-Belair, P. Jeandet, Langmuir 19, 5771 (2003)

(47) H. Ritacco, F. Kiefer, D. Langevin, Phys. Rev. Lett. 98, 244501 (2007)

(48) J.M. Boulton-Stone, J.R. Blake, J. Fluid Mech. 254, 437 (1993)

(49) A. Handa, A. N. Emery, R.E. Spier, Dev. Biol. Stand. 66, 241 (1987)

(50) K.T. Kunas, E.T. Papoutsakis, Biotech. Bioengng. 36, 476 (1990)

(51) T. G. Van Leeuwen, J.H. Meertens, E. Velema, M.J. Post, C. Borst, Circulation 87, $1258(1993)$

(52) E.A. Brujan, Europhys. Lett. 50, 175 (2000)

(53) N. Vandewalle, J.F. Lentz, S. Dorbolo, F. Brisbois, Phys. Rev. Lett. 86, 179 (2001)

(54) J. Herman, R. Mesler, J. Colloid Interface Sci. 117, 565 (1987)

(55) H.N. Oguz, A. Prosperetti, J. Fluid Mech. 203, 143 (1990)

(56) M.S. Longuet-Higgins, J. Fluid Mech. 214, 395 (1990)

(57) A. Prosperetti, H.N. Oguz, Ann. Rev. Fluid Mech. 25, 577 (1993) 
(58) M. Rein, J. Fluid Mech. 306, 145 (1996)

(59) D. Morton, M. Rudman, L. Jong-Leng, Phys. Fluids 12, 747 (2000)

(60) T.B. Benjamin, A.T Ellis, Philos. Trans. R. Soc. London, Ser. A 260, 221 (1966)

(61) G. Liger-Belair, Effervescence! La science du champagne (Odile Jacob, Paris, 2009) 


\section{FIGURE CAPTIONS}

Figure 1: Oblique view of the bubble monolayer composed of quite millimetric bubbles organized in a hexagonal pattern, each bubble being surrounded by an arrangement of six neighbors (a); schematic transversal representation of five aligned bubbles in touch in the monolayer (b).

Figure 2: Schematic representation, in a rupturing spherical bubble-cap with a radius of curvature $2 R$, of the growing hole surrounded by a rim which collects the liquid and propagates driven by surface tension forces.

Figure 3: Scheme of the two production ways of droplets from a bursting bubble; redrawn from the article by Resch et al. ${ }^{19}$

Figure 4: High-speed photographs showing two cavities left by floating bubbles after the disintegration of the bubble-cap (bar $=1 \mathrm{~mm})$.

Figure 5: Reconstructed time-sequence showing the situation following the collapse of a single millimetric bubble; the collapse of the empty cavity (frame 1) leads to the projection of a tiny liquid jet (frames 2 and 3) which breaks up into several jet drops (frame 4) (bar = 1 $\mathrm{mm})$.

Figure 6: Comparison between the Rayleigh-Plateau instability which develops along the tiny champagne jet (a), and the one which develops along a cylindrical water jet falling from a leaking faucet (b) (C Gérard Liger-Belair / Daniel Schwen). 
Figure 7: Time sequence (taken through a high-speed video camera filming 7000 frames per second) showing the collapse of a millimetric champagne bubble, the formation of the Worthington jet, and the development of the Rayleigh-Plateau instability along the jet; time in ms appears close to the corresponding frame.

Figure 8: Time sequence showing the bouncing of a tiny droplet on the champagne surface, and its coalescence with a smaller one (see the detail below); time in ms appears close to the corresponding frame.

Figure 9: The collapse of hundreds of bubbles at the free surface radiate a cloud of tiny droplets which is characteristic of champagne and other sparkling wines and which complements the sensual experience of the taster (ㄷ Alain Cornu / Collection CIVC).

Figure 10: The aerosol constituted by myriads of tiny droplets ejected from bubbles' bursting above the surface of a coupe, as seen through laser tomography technique; the droplets' trajectories are materialised by blue streaks of light during the 1s-exposure time of a digital photo camera. (Photograph by G. Liger-Belair, F. Beaumont and G. Polidori).

Figure 11: Concentration factors analysis of all masses present in the mass spectra of champagne aerosols and bulk, respectively, in the whole mass range m/z 150-1000; more details are provided in Liger-Belair et al. ${ }^{44}$

Figure 12: A few seconds after pouring, and after the collapse of the foamy head, the surface of a flute is covered with a layer of quite mono disperse millimetric bubbles, where bubbles 
arrange themselves in an approximate hexagonal pattern, strikingly resembling those in beeswax $($ bar $=1 \mathrm{~cm})$.

Figure 13: Flower-shaped structure, as frozen through high-speed photography, found during the collapse of bubbles in the bubble raft at the free surface of a flute poured with champagne $($ bar $=1 \mathrm{~cm})$

Figure 14: Shear stresses experienced by bubbles adjacent to a collapsing one at the free surface of a flute poured with champagne $(\mathrm{bar}=1 \mathrm{~cm})$.

Figure 15: Schematic transversal representation of the situation, as frozen after the disintegration of the central bubble-cap.

Figure 16: Time sequence showing the dynamics of adjoining bubbles in touch with a collapsing one at the free surface of flute poured with champagne; the whole process was filmed at 1500 frames/s; from frame 4, in the center of the empty cavity left by the collapsing bubble, a tiny air-bubble entrapment is observed (bar $=1 \mathrm{~mm})$.

Figure 17: Comparison, as it divides into jet drops due to the Rayleigh-Plateau instability, between the jet which follows the collapse of a bubble in the bubble raft (a), and that which follows a single bubble collapse (b).

Figure 18: Time-sequence, filmed at 5000 frames/s, showing the flower-shaped structure taken by bubbles neighboring a collapsing one, and formation of the subsequent Worthington jet. 
Figure 19: Time-sequence, filmed at 5000 frames/s, showing the formation of a largely tilted Worthington jet as the neighbouring bubbles' symmetry is broken around a collapsing bubble.

Figure 20: Close-up on the tilted Worthington jet (frozen through high-speed photography) as the neighbouring bubbles' symmetry is broken around a collapsing one (bar $=1 \mathrm{~mm})$.

Figure 21: Time-sequence showing a capillary wave travelling radially after the collapse of a champagne bubble (frame 1); as the wave reaches distant bubbles, their synchronous explosion is observed, as if the wave had triggered the bubble rupture; moreover, it is worth noting that the Worthington jets resulting from the "wave-driven" bubbles collapse are tilted towards the initial collapsing bubble (frame 8). 


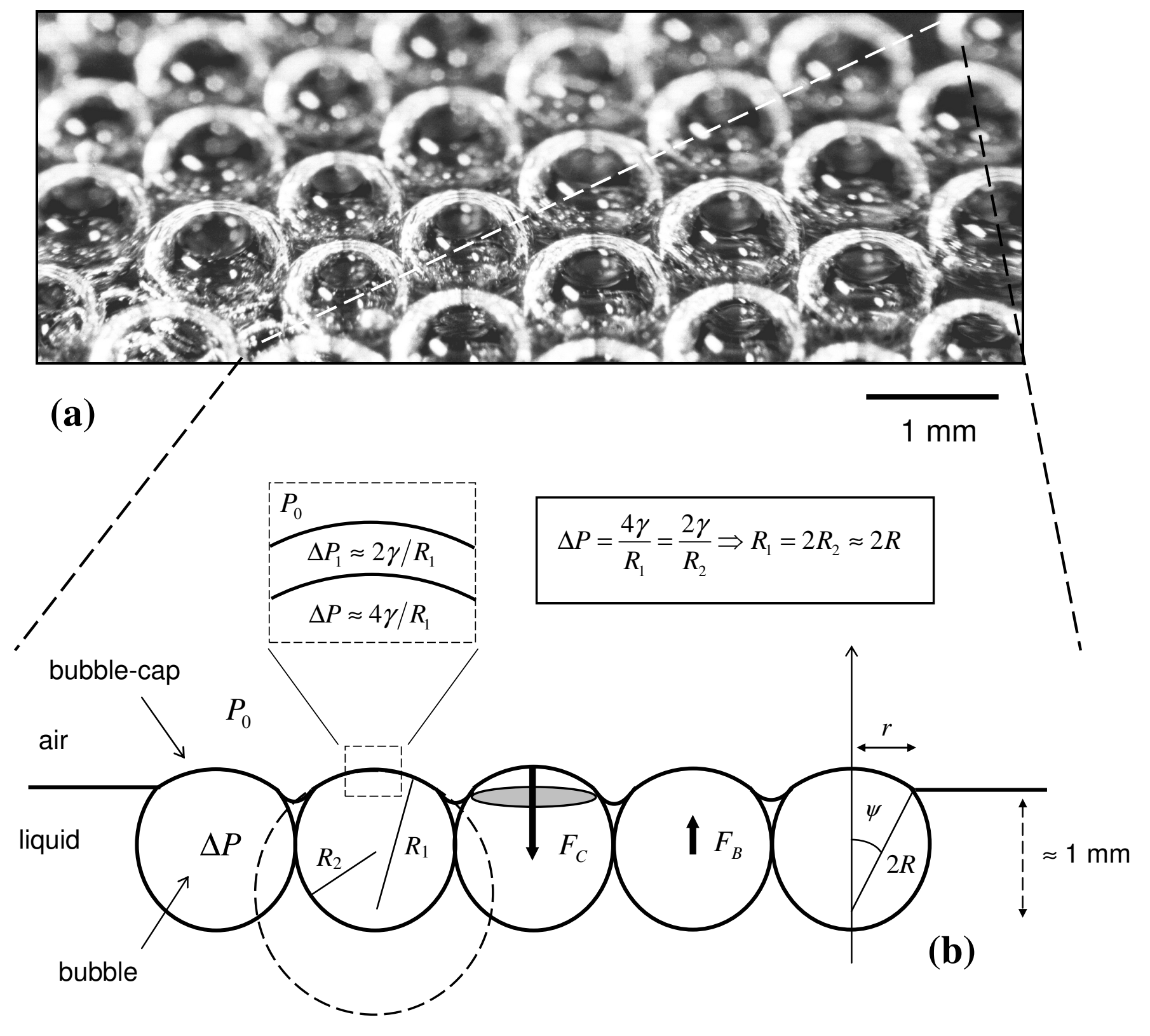

Figure 1 


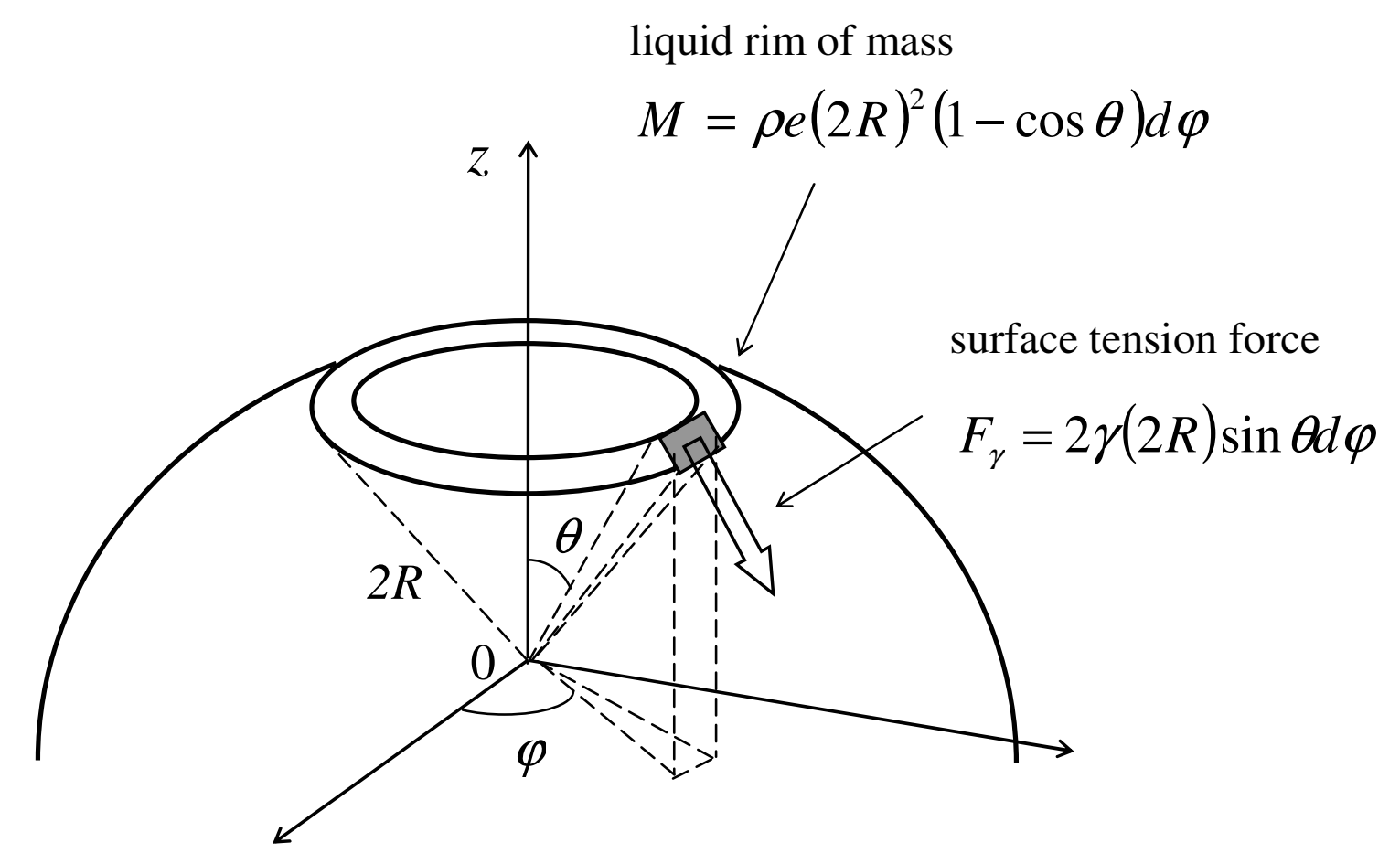

Figure 2 


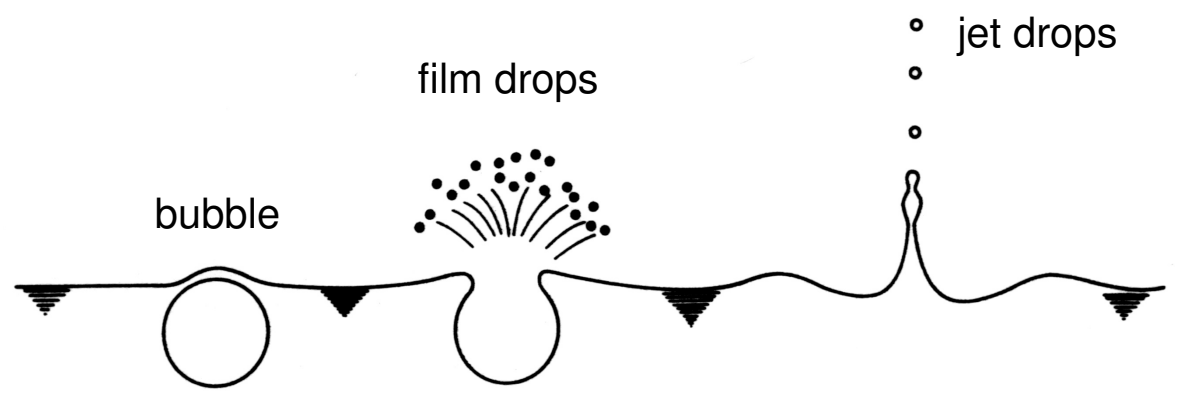

Figure 3 

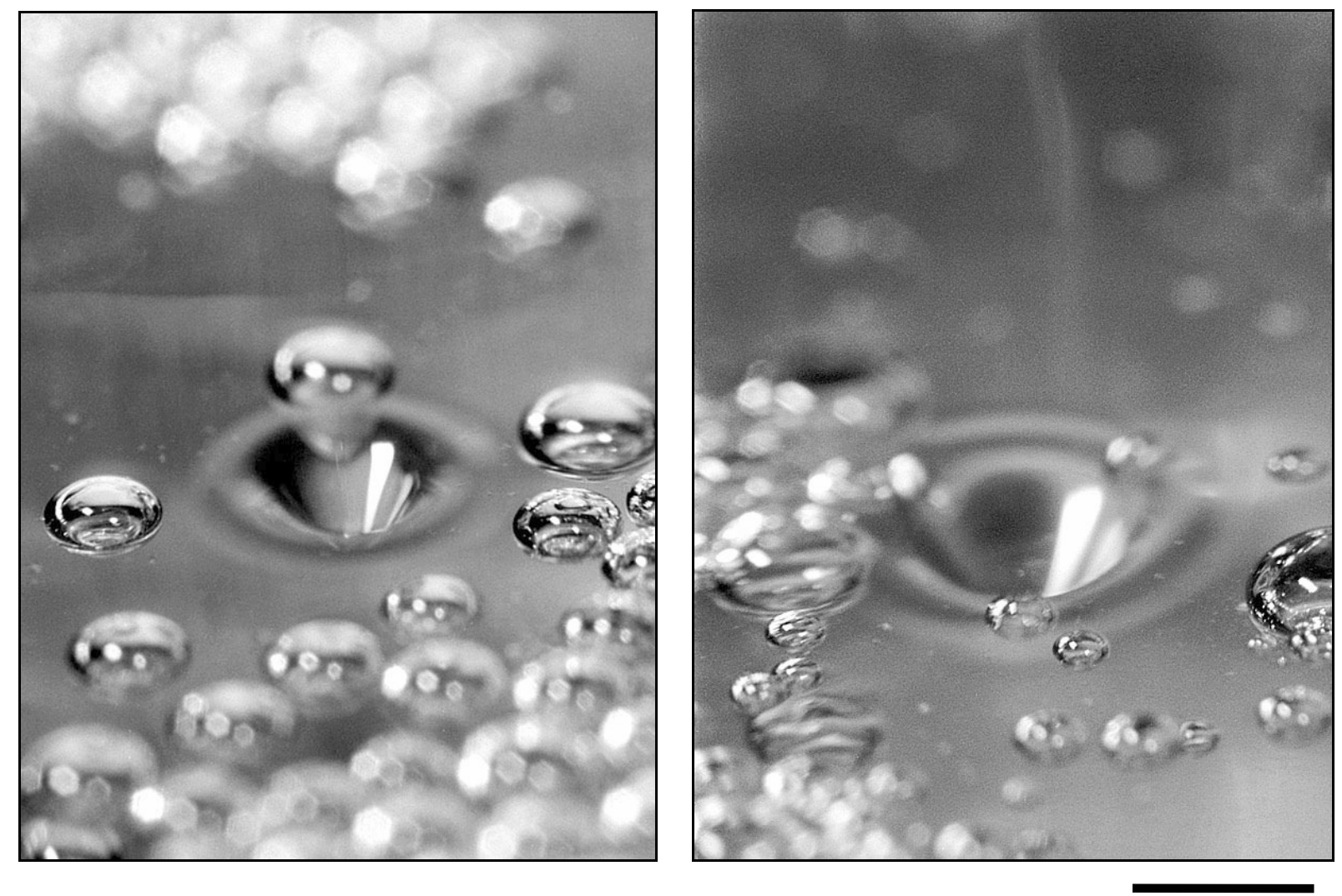

Figure 4 

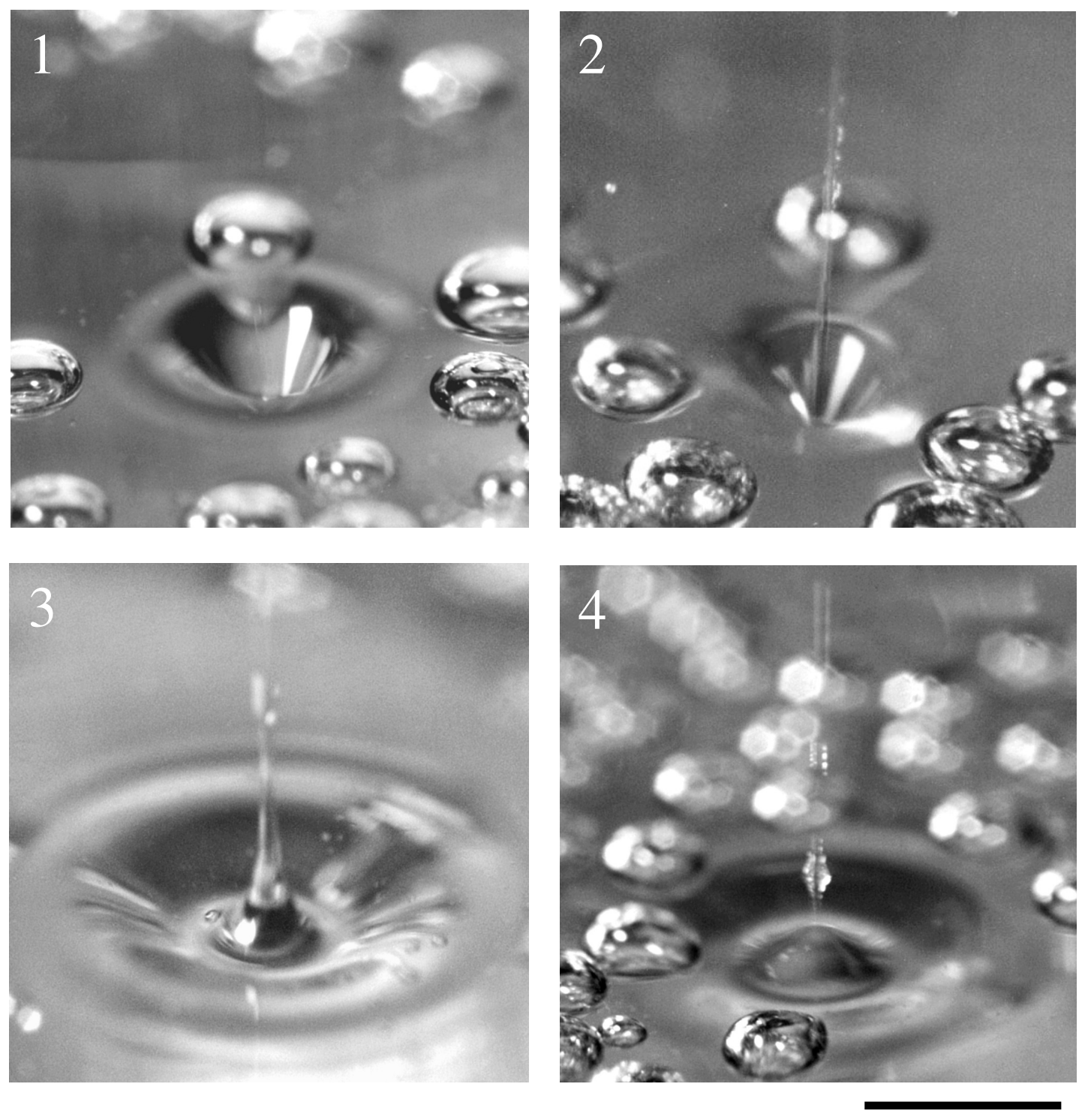

Figure 5 

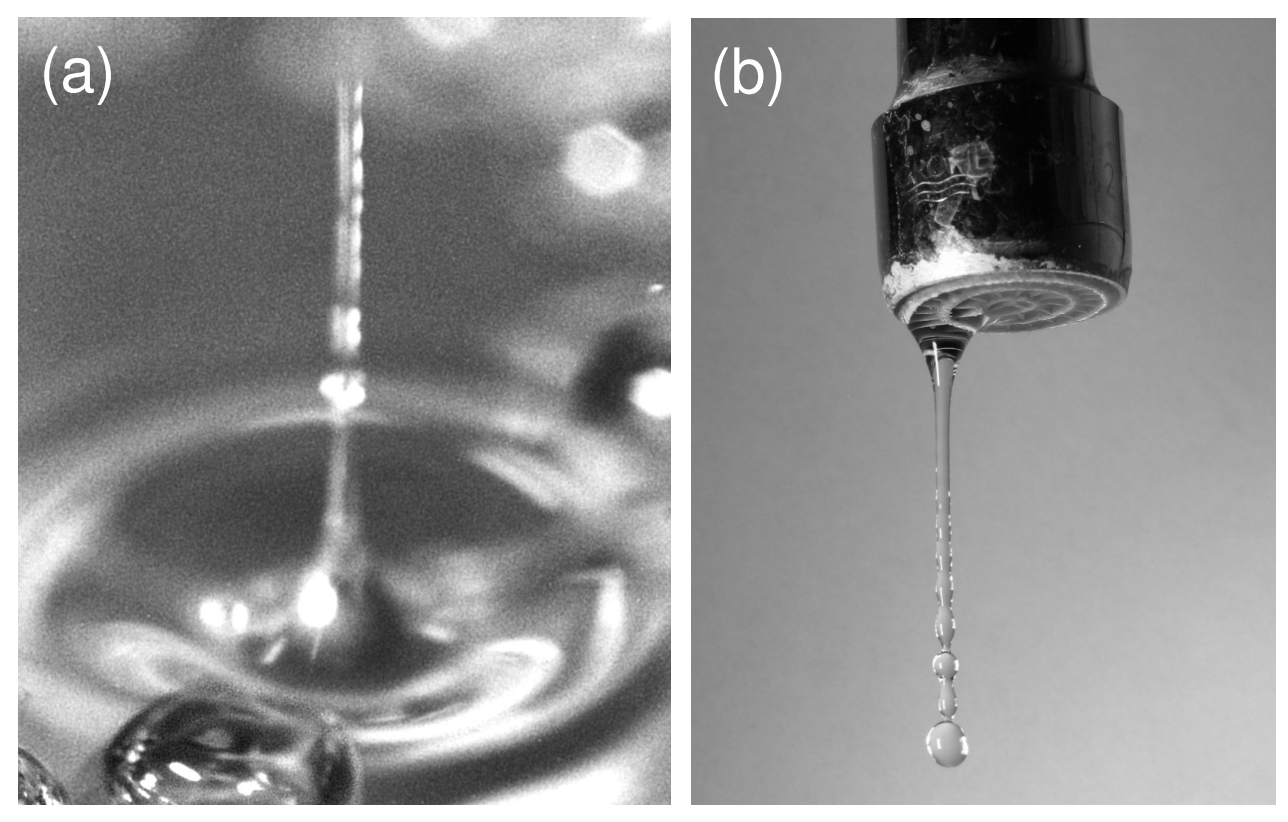

Figure 6 


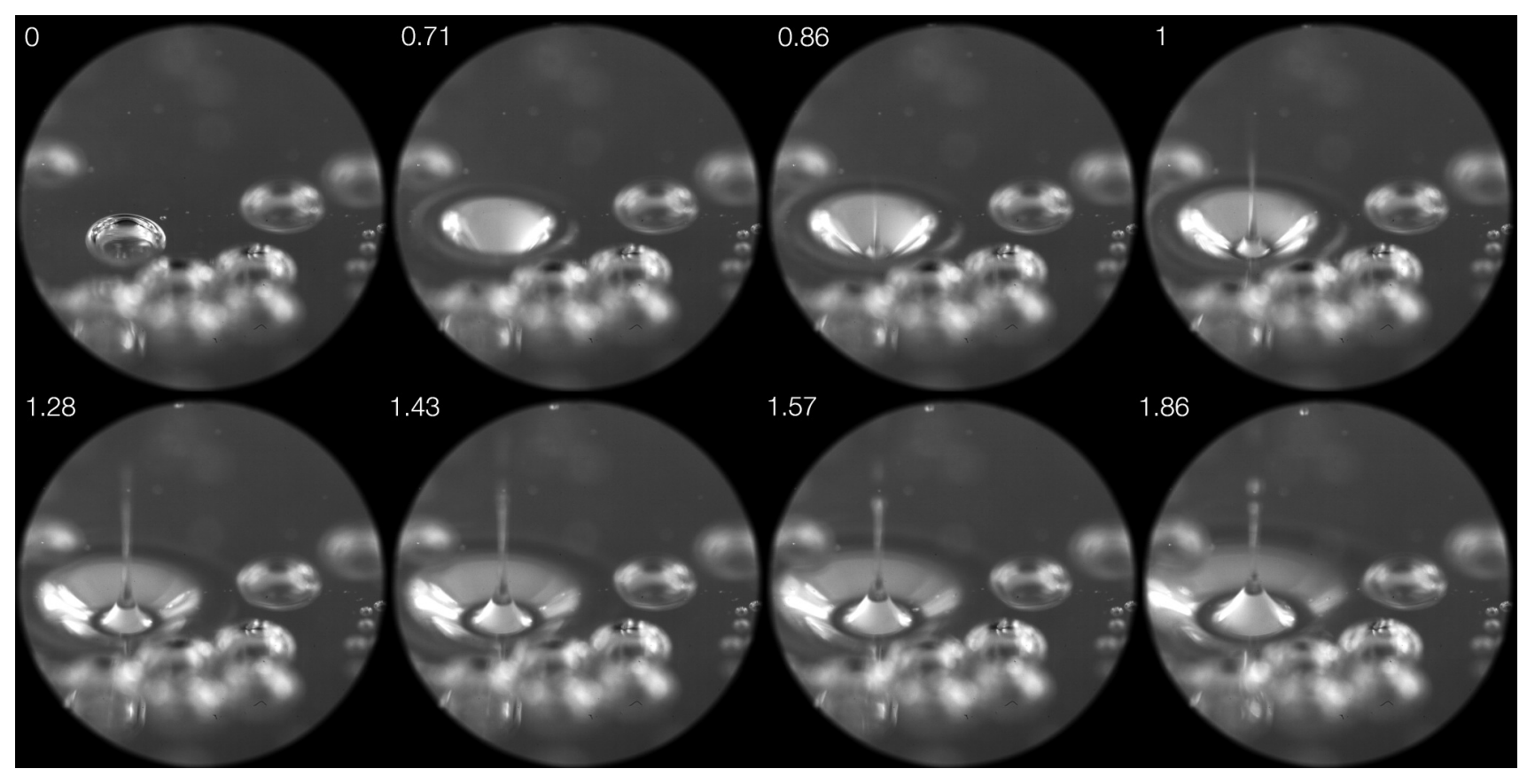

Figure 7 

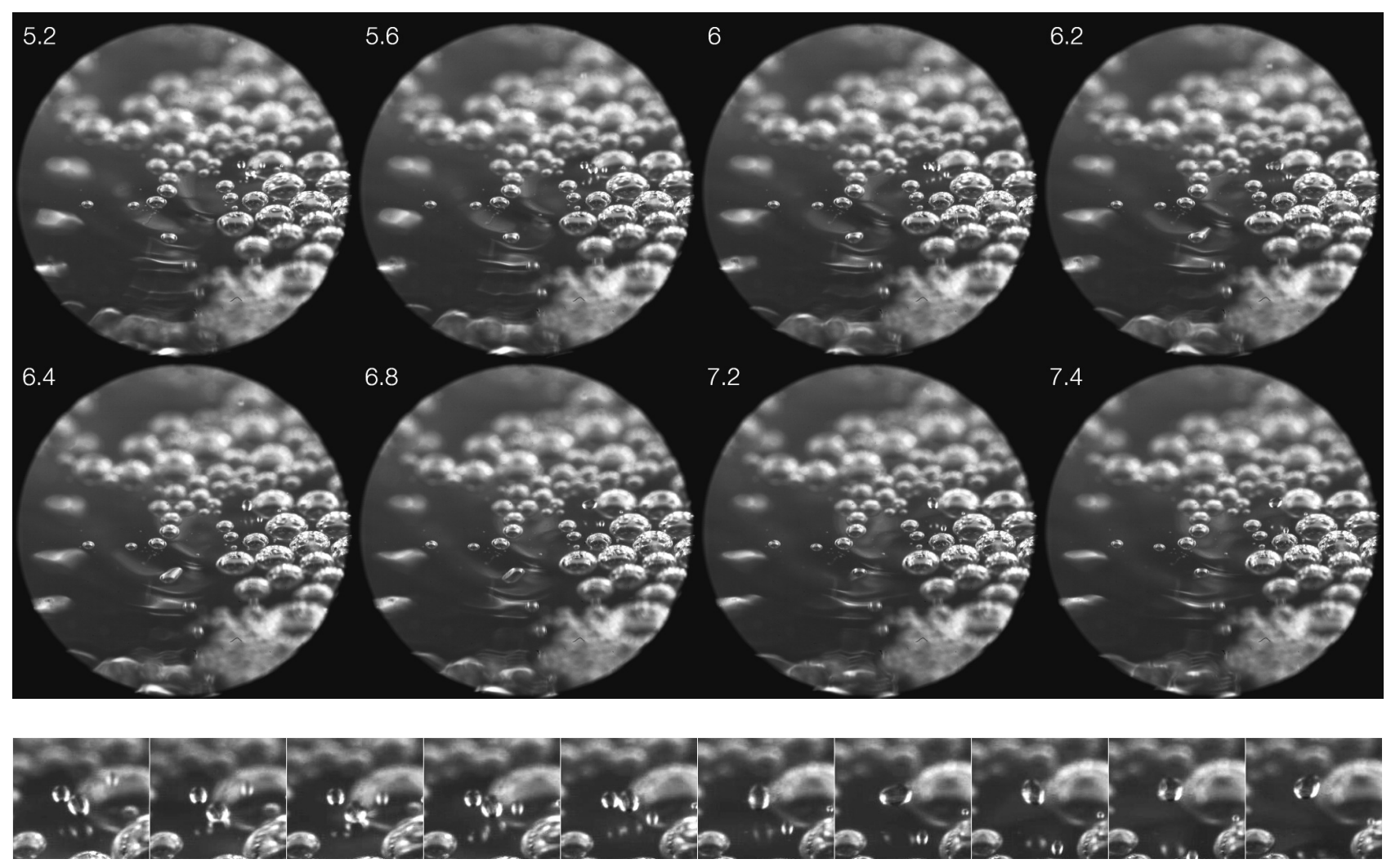

Figure 8 


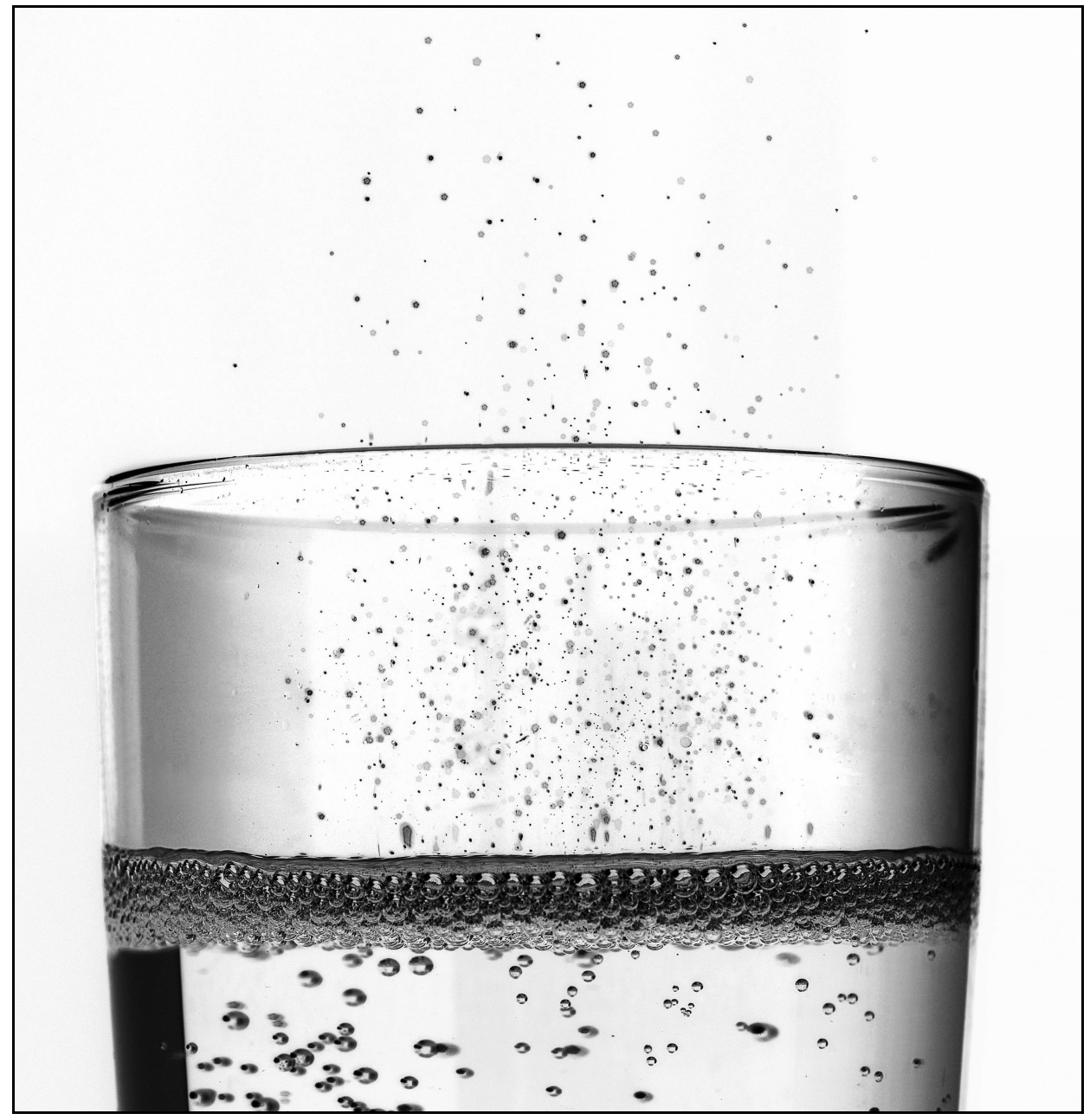

Figure 9 


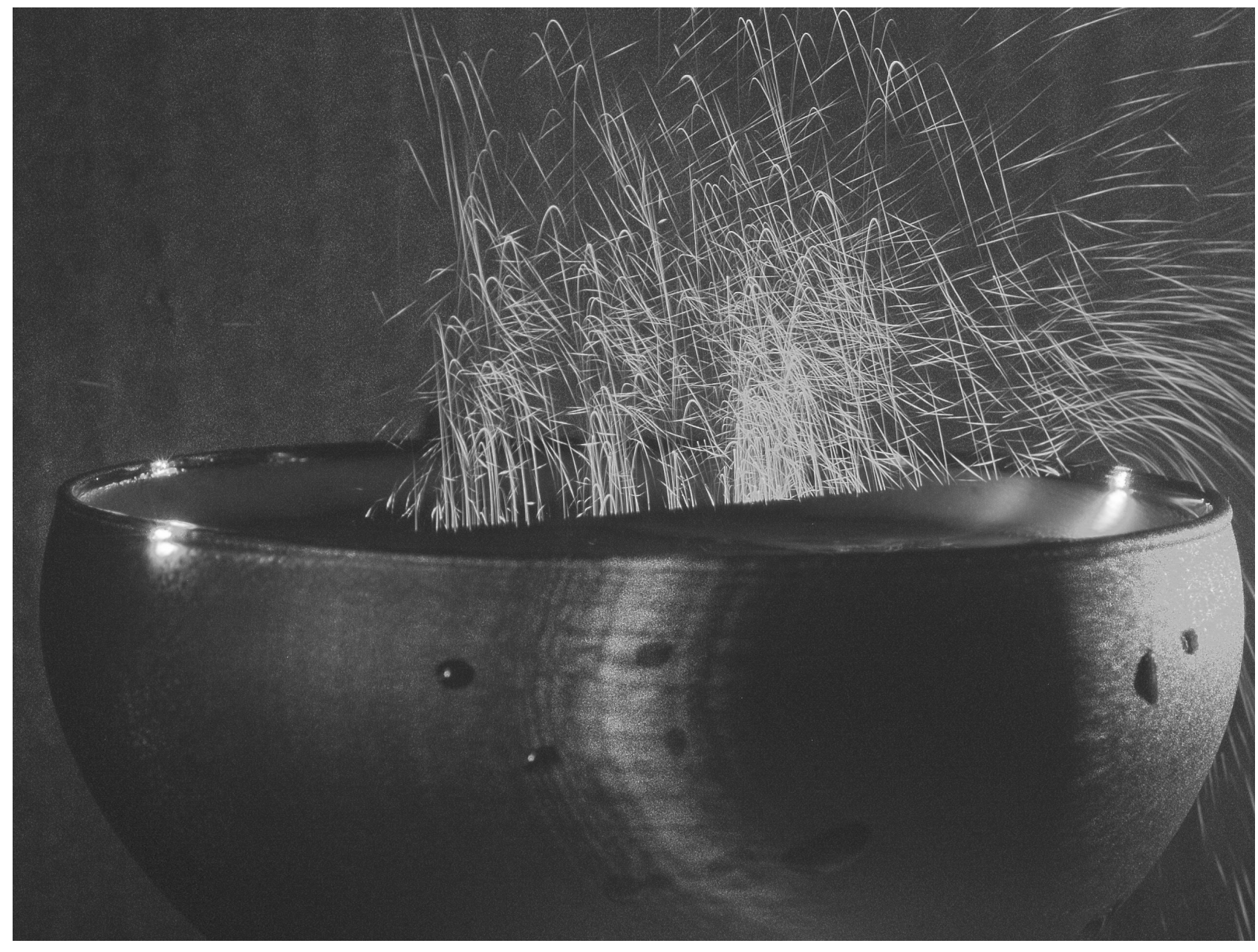

Figure 10 


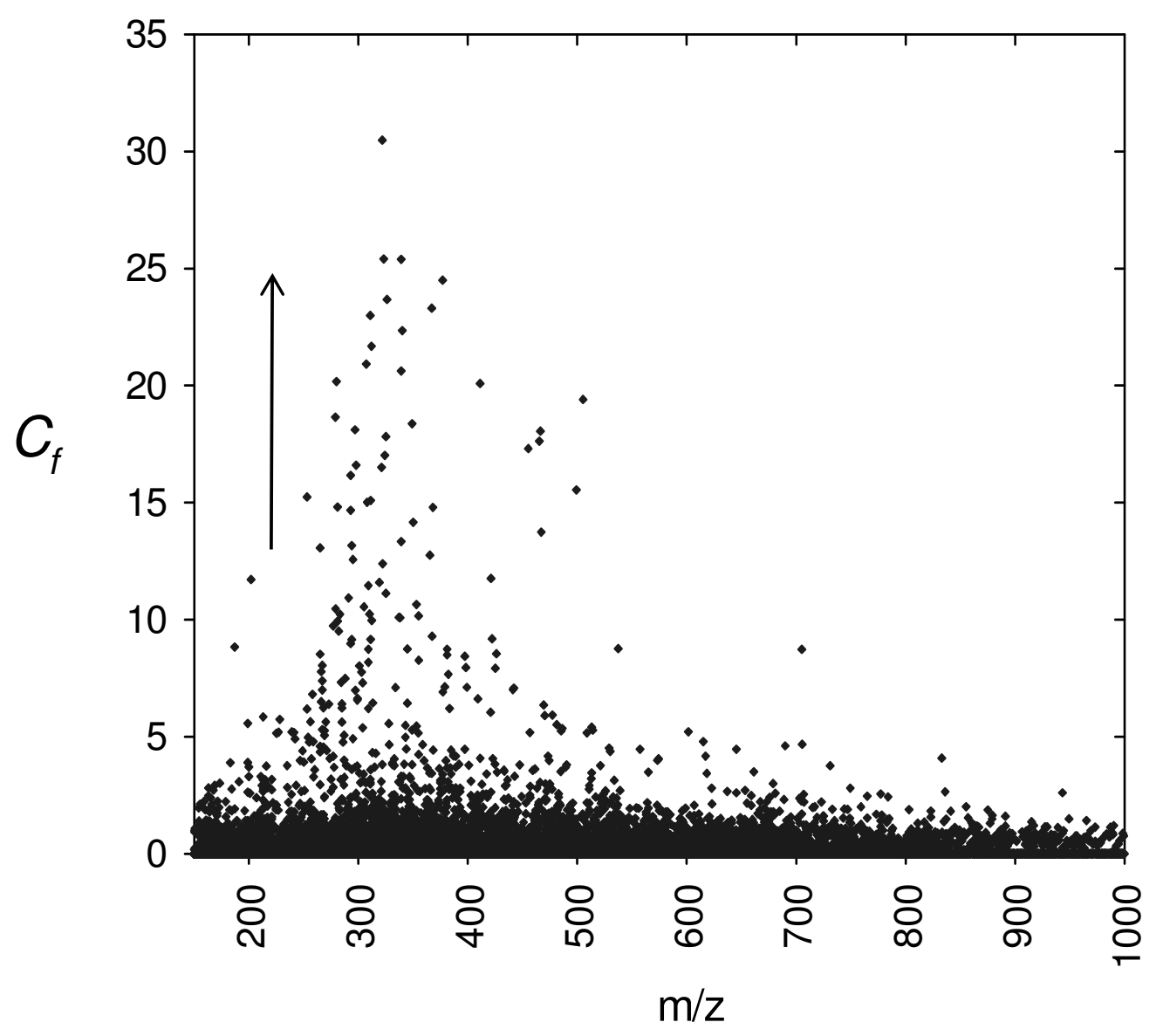

Figure 11 


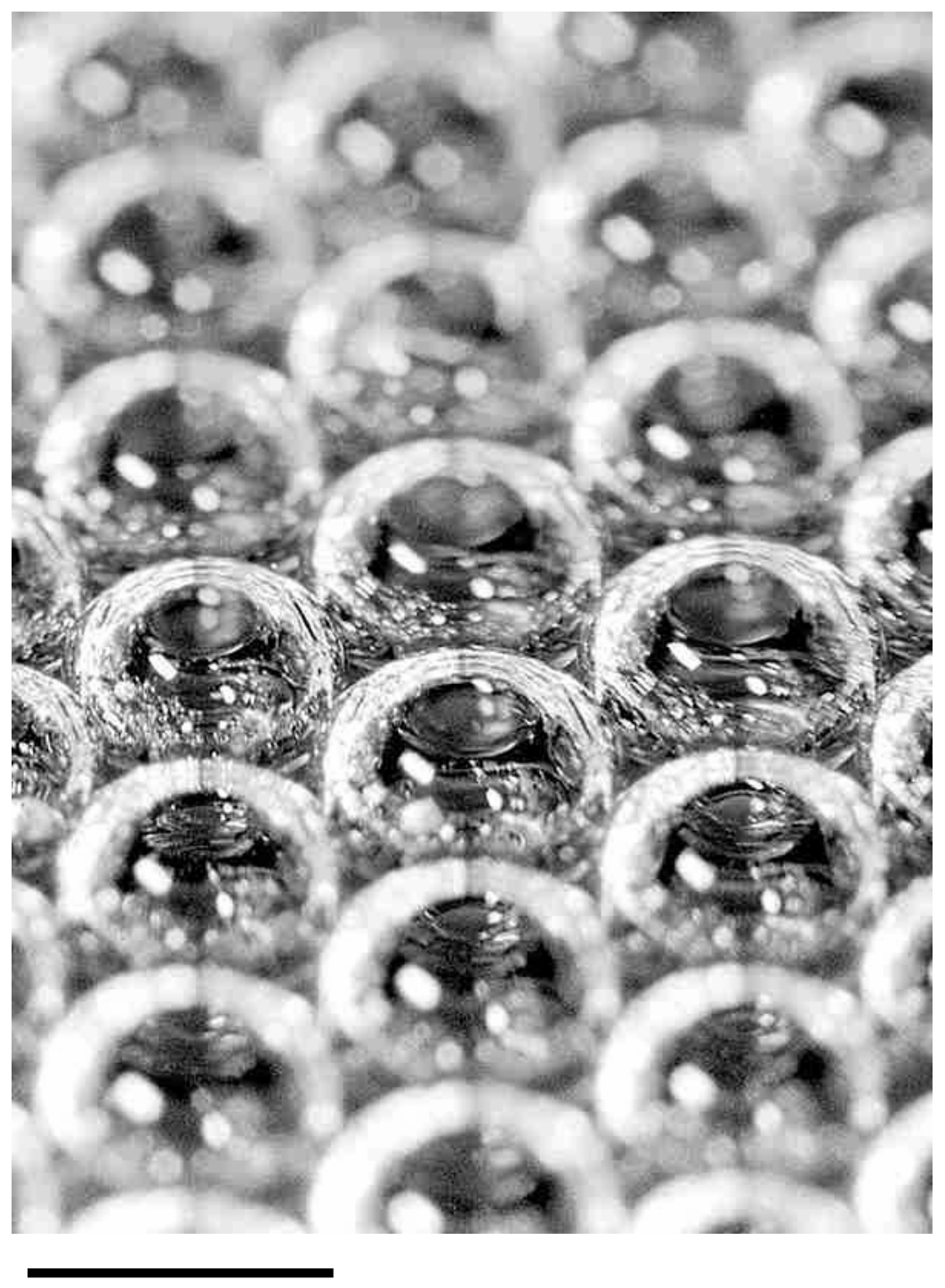

Figure 12 


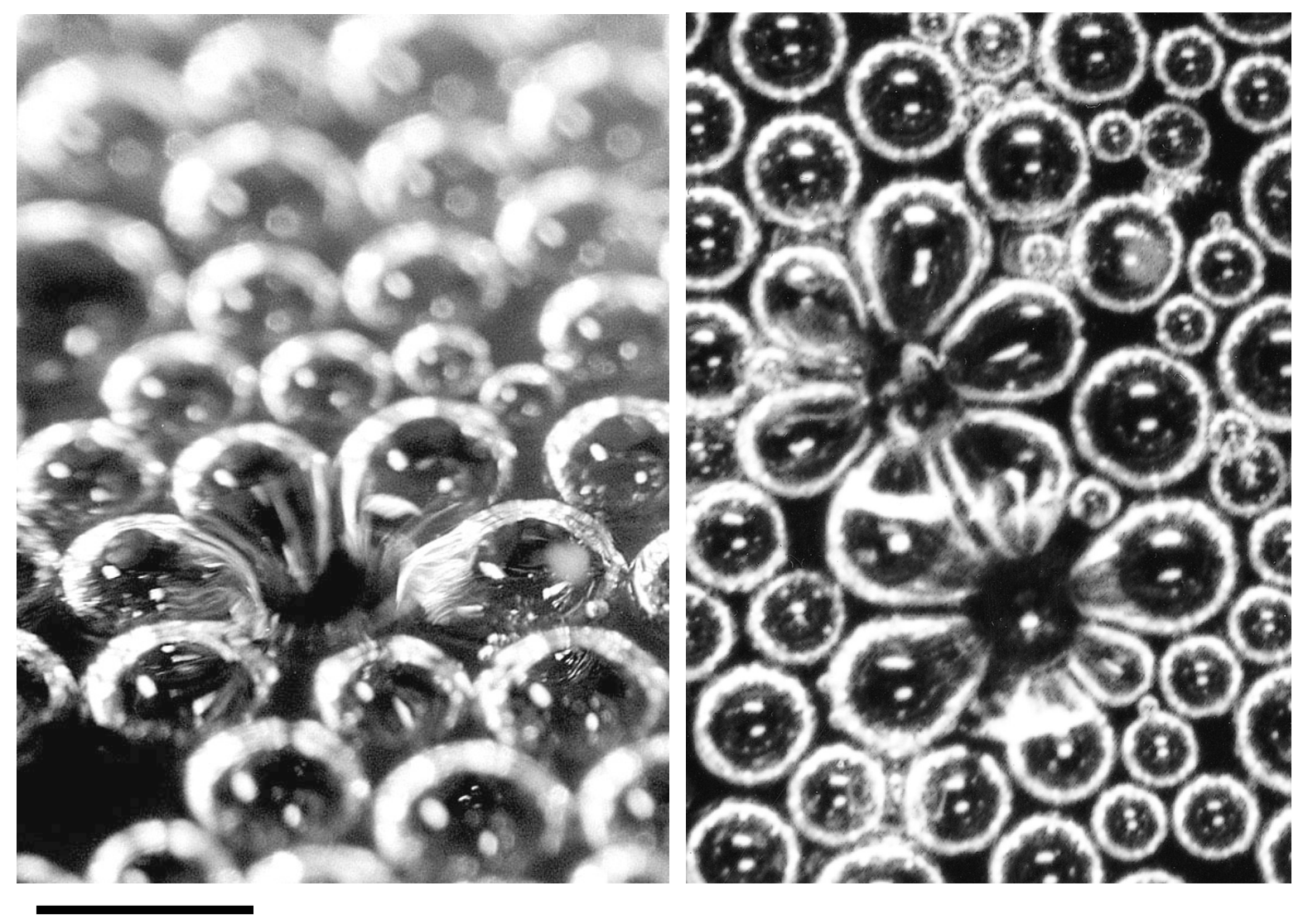

Figure 13 


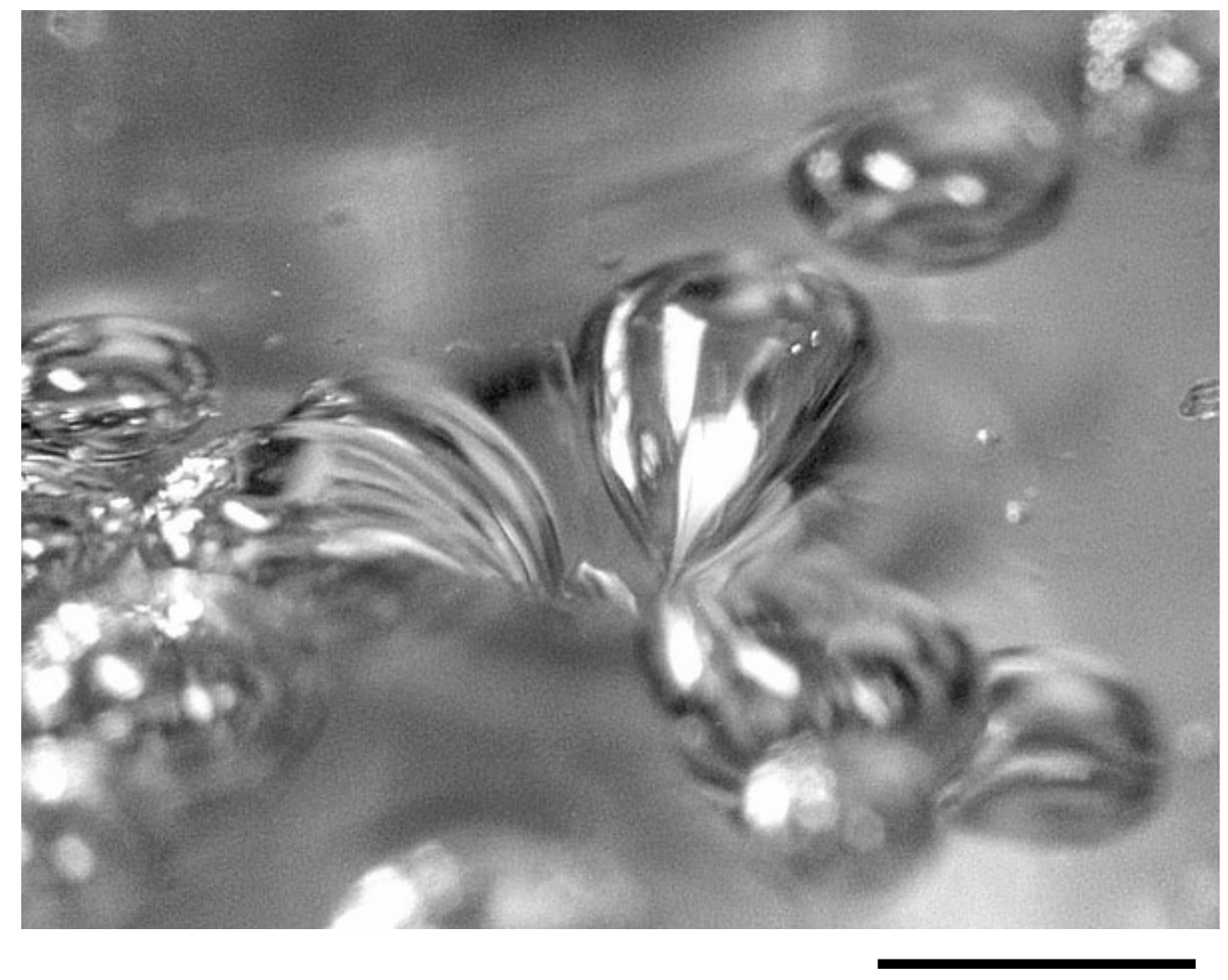

Figure 14 


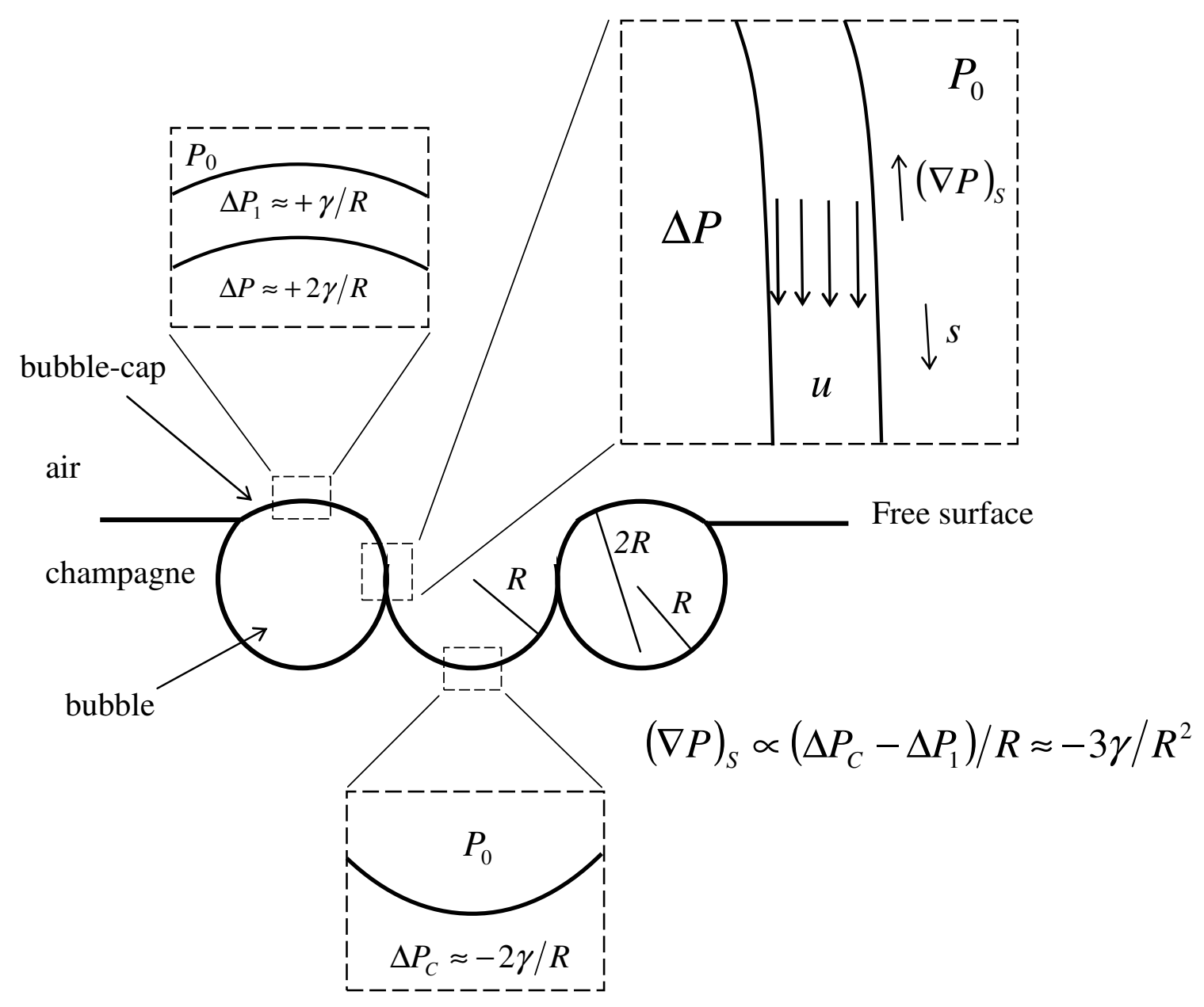

Figure 15 
This bubble-cap is about to rupture
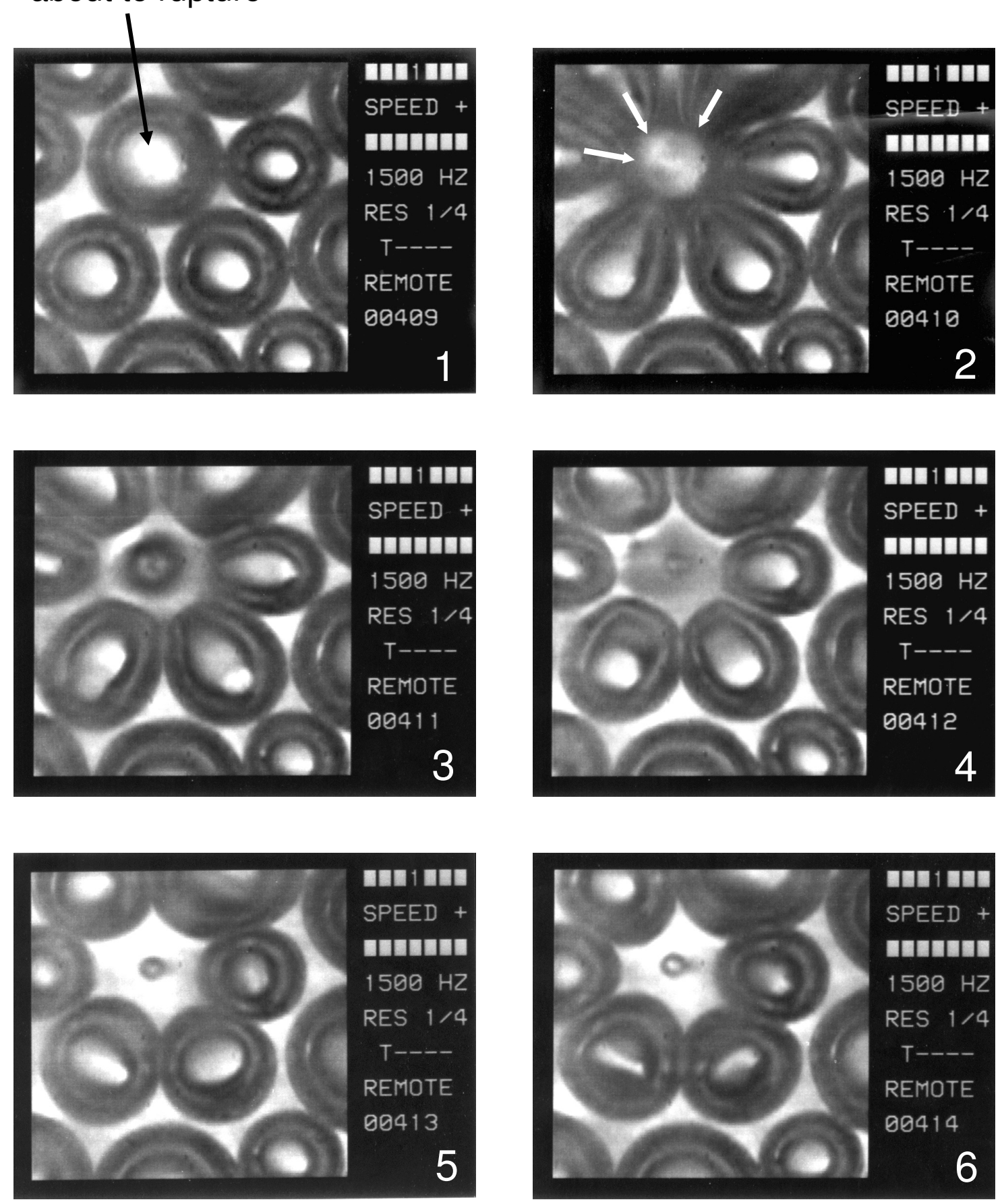

Figure 16 

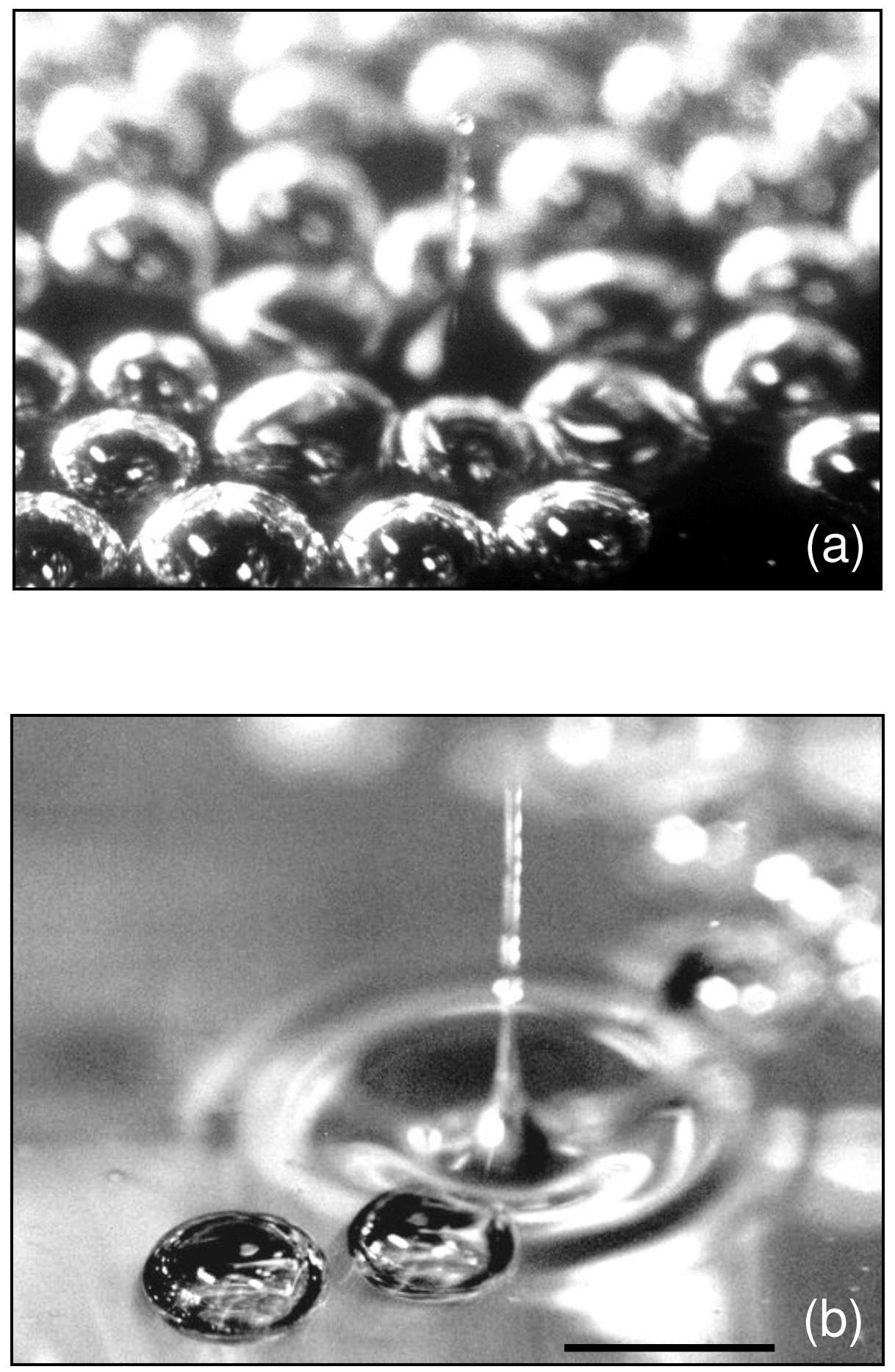

Figure 17 


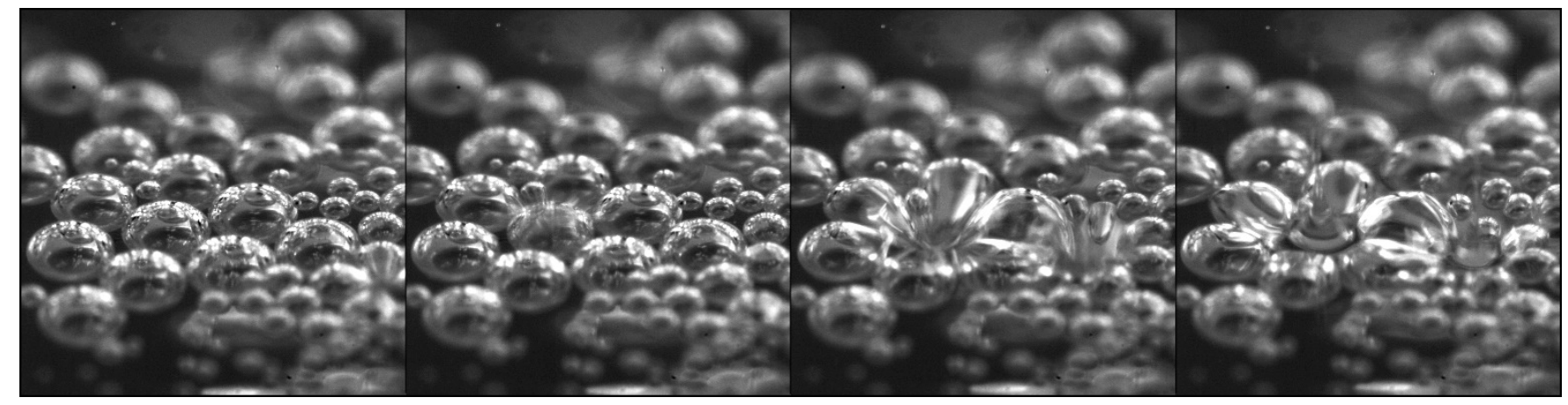

Figure 18 


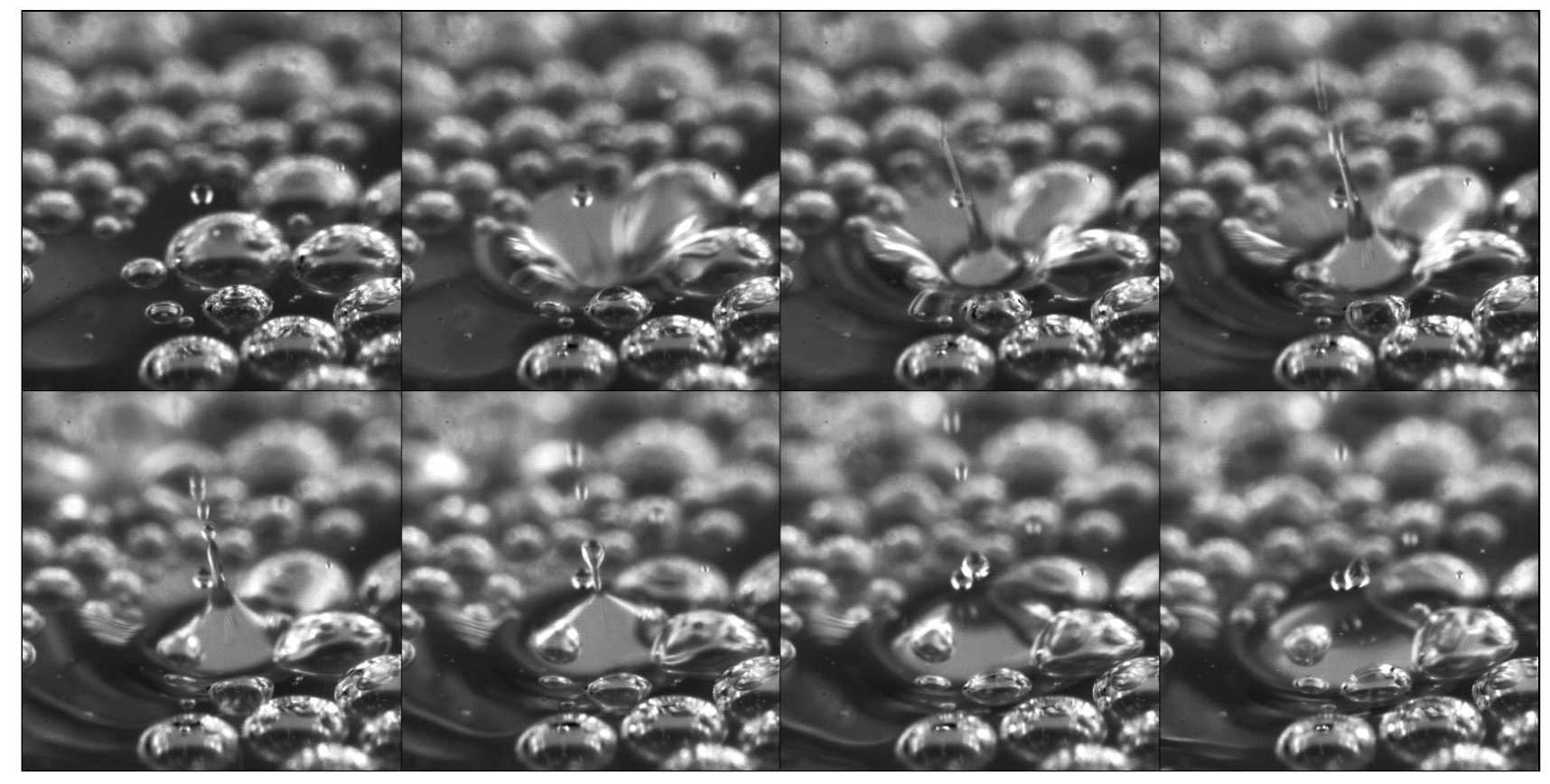

Figure 19 


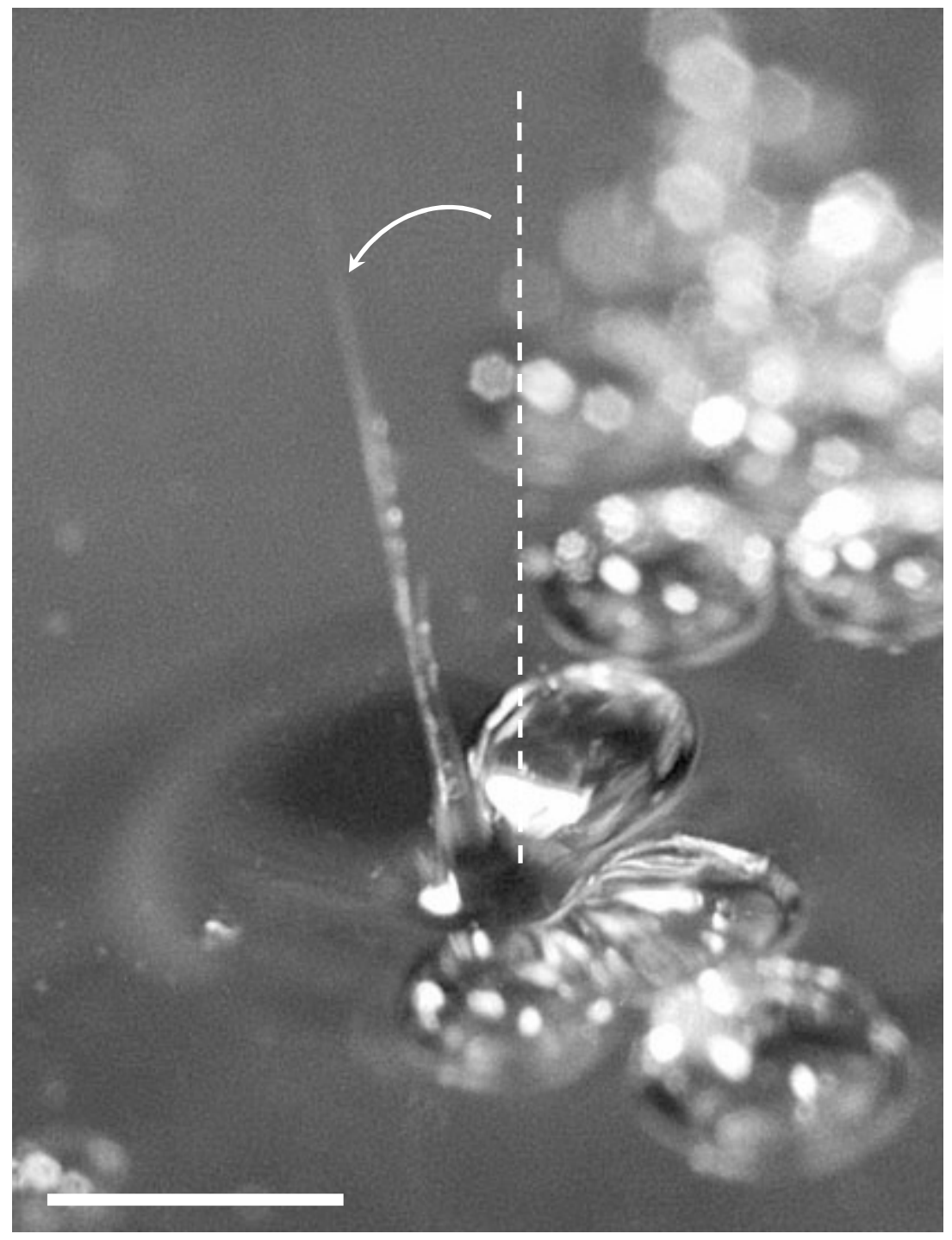

Figure 20 


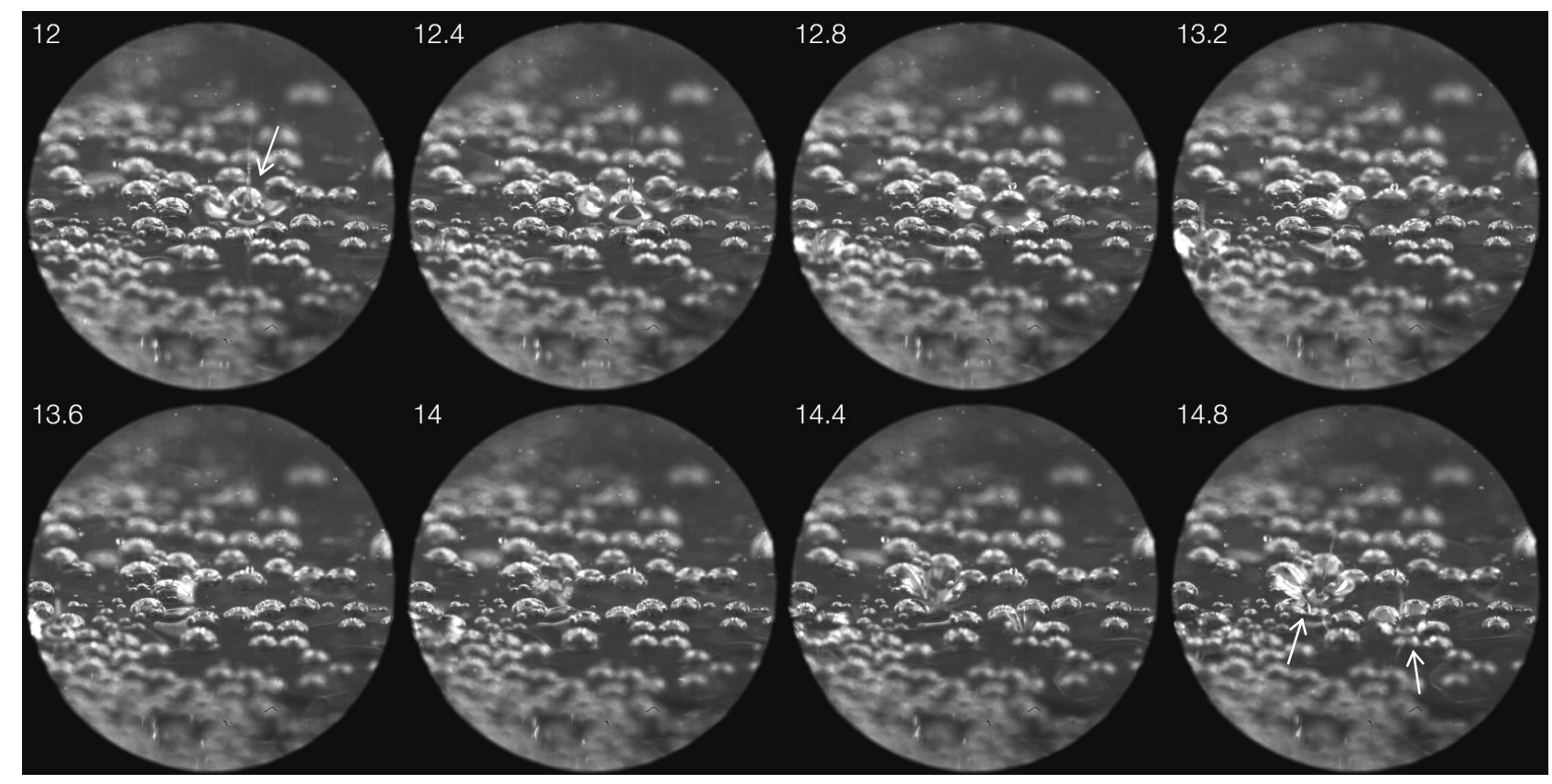

Figure 21 